\title{
Astrospheres and Solar-like Stellar Winds
}

\author{
Brian E. Wood \\ JILA, University of Colorado \\ 440 UCB, Boulder, CO 80309-0440 \\ email: woodb@origins.colorado.edu \\ http://origins. colorado. edu/ $\sim_{\text {woodb }}$

\section{Living Reviews in Solar Physics ISSN 1614-4961}

Accepted on 30 June 2004

Published on 29 July 2004

(Revised on 13 July 2007)

\begin{abstract}
Stellar analogs for the solar wind have proven to be frustratingly difficult to detect directly. However, these stellar winds can be studied indirectly by observing the interaction regions carved out by the collisions between these winds and the interstellar medium (ISM). These interaction regions are called "astrospheres", analogous to the "heliosphere" surrounding the Sun. The heliosphere and astrospheres contain a population of hydrogen heated by charge exchange processes that can produce enough H I Ly $\alpha$ absorption to be detectable in UV spectra of nearby stars from the Hubble Space Telescope (HST). The amount of astrospheric absorption is a diagnostic for the strength of the stellar wind, so these observations have provided the first measurements of solar-like stellar winds. Results from these stellar wind studies and their implications for our understanding of the solar wind are reviewed here. Of particular interest are results concerning the past history of the solar wind and its impact on planetary atmospheres.
\end{abstract}

This review is licensed under a Creative Commons Attribution-Non-Commercial-NoDerivs 2.0 Germany License. http://creativecommons.org/licenses/by-nc-nd/2.0/de/ 


\section{Imprint / Terms of Use}

Living Reviews in Solar Physics are published by the Max Planck Institute for Solar System Research, Max-Planck-Str. 2, 37191 Katlenburg-Lindau, Germany. ISSN 1614-4961

This review is licensed under a Creative Commons Attribution-Non-Commercial-NoDerivs 2.0 Germany License: http://creativecommons.org/licenses/by-nc-nd/2.0/de/

Because a Living Reviews article can evolve over time, we recommend to cite the article as follows:

Brian E. Wood,

"Astrospheres and Solar-like Stellar Winds",

Living Rev. Solar Phys., 1, (2004), 2. [Online Article]: cited [<date $>$ ], http://www.livingreviews.org/lrsp-2004-2

The date given as $<$ date $>$ then uniquely identifies the version of the article you are referring to. 


\section{Article Revisions}

Living Reviews supports two different ways to keep its articles up-to-date:

Fast-track revision A fast-track revision provides the author with the opportunity to add short notices of current research results, trends and developments, or important publications to the article. A fast-track revision is refereed by the responsible subject editor. If an article has undergone a fast-track revision, a summary of changes will be listed here.

Major update A major update will include substantial changes and additions and is subject to full external refereeing. It is published with a new publication number.

For detailed documentation of an article's evolution, please refer always to the history document of the article's online version at http://www.livingreviews.org/lrsp-2004-2.

13 July 2007: This revision includes 23 new references, two new Figures 11 and 13, and two revised Figures 14 and 15, which replace former Figures 12 and 13 of the original publication. Changes have been made to Sections 2.1, 2.3, 4.2, 5.2, 5.3 and 5.4. The most substantial revisions have taken place in Sections 4.3 and 5.1. Section 6, Conclusions, has been added.

See below for more details on the changes.

Page 9: I now acknowledge the early solar wind detection of the Soviet Luna missions of 1959. Added reference to Cranmer (2002).

Page 13: I have reworded the third paragraph to take into account Voyager 1's 2004 crossing of the termination shock.

Page 20: In Section 4.2, I have revised the third paragraph to take into account numerous new heliospheric absorption detections that have been made since 2004. I have also revised the text below to consider the results of more sophisticated heliospheric models that have been published in the past few years. Added references to Malama et al. (2006), Wood et al. (2007b), Opher et al. (2006), Izmodenov et al. (2005), Pogorelov et al. (2006), and Wood et al. (2007a).

Page 23: This is where the most substantial revisions have taken place, in order to take into account the increase in astrospheric absorption detections from 6 to 13. This enlarges Table 1 and results in the addition of the two new Figures 11 and 13. The discussion of the wind measurements, and the inferred wind/activity and wind/age relations, is necessarily revised significantly, basically along the lines of Wood et al. (2005a). The recent work of Holzwarth and Jardine (2007) is also noted.

Page 31: Along with Section 4.3, this is where the most substantial revisions have taken place, in order to take into account the increase in astrospheric absorption detections from 6 to 13 . Figures 14 and 15 have been revised. The discussion of the wind measurements, and the inferred wind/activity and wind/age relations, is necessarily revised significantly, basically along the lines of Wood et al. (2005a). Added references to Schmitt and Liefke (2004), Strassmeier (2002), Schrijver and Title (2001), Toner and Gray (1988), and Petit et al. (2005).

Page 33: Some minor quantitative adjustments have had to be made based on the changes in Section 5.1, but there are no serious textual changes. In detail, $1.00 \pm 0.26$ was changed to $1.17 \pm 0.28$ in Equation 7, and the upper limit for $m=0-2$ from $\alpha<-1.0$ to $\alpha<-1.3$, for $m=0-1$ from $\alpha<-1.2$ to $\alpha<-1.7$. 
Page 35: In the second paragraph, $0.1 \%$ was changed to $0.2 \%$ and a reference to Minton and Malhotra (2007) added.

Page 35: The final paragraph has been revised to discuss the importance of solar-like winds for the atmospheric evolution of extrasolar planets, especially ones that orbit very close to their stars. Added references to Ribas et al. (2005), Grießmeier et al. (2004), and Preusse et al. (2005).

Page 36: Section 6 has been added to briefly discuss the future of the subject, and to provide a less abrupt ending to the article. 


\section{Contents}

1 Introduction $r$

2 Background Material $\quad 9$

2.1 The solar wind . . . . . . . . . . . . . . . . . . . . . . . . . . . . . . . . . .

2.2 The local interstellar medium . . . . . . . . . . . . . . . . . . . . . . . . . . . . . . . . . . . . . . . . .

2.3 The structure of the heliosphere . . . . . . . . . . . . . . . . 12

3 Direct Wind Detection Techniques $r$

4 Detecting Winds Through Astrospheric Absorption 16

4.1 Analyzing H I Lyman-alpha lines . . . . . . . . . . . . . . . . . . . . . . 16

4.2 Comparing heliospheric absorption with model predictions . . . . . . . . . . . . 20

4.3 Measuring stellar mass loss rates . . . . . . . . . . . . . . . . . . . . 23

5 Implications for the Sun and Solar System $\quad 31$

5.1 Inferring the mass loss history of the Sun . . . . . . . . . . . . . . . . 31

5.2 Magnetic braking . . . . . . . . . . . . . . . . . . . . . . 33

5.3 The Faint Young Sun problem . . . . . . . . . . . . . . . . . . . . 34

5.4 Erosion of planetary atmospheres . . . . . . . . . . . . . . . . . 35

6 Conclusions $\quad 36$

$\begin{array}{llr}7 & \text { Acknowledgements } & 37\end{array}$

$\begin{array}{lr}\text { References } & 38\end{array}$ 



\section{Introduction}

It goes without saying that it is generally much easier to study the nearby Sun than it is to study much more distant solar-like stars. Nevertheless, stellar research can address questions about the Sun that observations of the Sun alone cannot answer. The Sun only provides one example of a cool main sequence star, so it cannot tell us by itself how its various properties relate to each other. By observing other solar-like stars, we can see how properties such as stellar activity, rotation, and age are correlated. This can teach us a lot about why the Sun has the properties it does today. It can also tell us what the Sun was like in the past and what it will be like in the future.

Many stellar analogs of solar phenomena are available for study: photospheres, chromospheres, coronae, starspots, magnetic fields, rotation, asteroseismology, etc. (see Skumanich, 1972; Linsky, 1980; Vogt et al., 1987; Gustafsson and Jørgensen, 1994; Johns-Krull and Valenti, 1996; Christensen-Dalsgaard, 2003; Favata and Micela, 2003; Güdel, 2004). Comparing solar properties with those observed for other stars provides a useful context for the solar measurements, improving our understanding of the Sun as well as for stars in general. However, one major solar phenomenon that has proven to be very difficult to study for other stars is the solar wind.

Some types of stellar winds are very easy to detect and study spectroscopically. The massive, radiation-pressure driven winds of hot stars and the cool, massive winds of red giants and supergiants both produce P Cygni emission line profiles that allow the measurement of wind properties with reasonable precision (Harper et al., 1995; Mullan et al., 1998; Kudritzki and Puls, 2000). However, these stars are not solar-like and the winds of these stars are not analogous to the much weaker wind that we see emanating from the Sun. The weak and fully ionized solar wind provides no spectral diagnostics analogous to those used to study more massive stellar winds. Directly detecting a truly solar-like wind around another solar-like star has therefore proven to be a formidable problem.

The first clear detections of winds around other solar-like stars have come from UV spectra of nearby stars from the Hubble Space Telescope (HST). Stellar H I Ly $\alpha$ lines at $1216 \AA$ are always contaminated by very broad, saturated H I absorption. For a long time, this absorption was assumed to be entirely from interstellar H I. However, for some of the nearest stars, the interstellar medium (ISM) cannot account for all of the observed absorption. With the assistance of complex hydrodynamic models of the solar wind/ISM interaction, the excess Ly $\alpha$ absorption has been convincingly identified as being partly due to heated H I gas within our own heliosphere and partly due to analogous H I gas within the "astrospheres" surrounding the observed stars. Note that the word "astrosphere" is used here as the stellar analog for "heliosphere", although "asterosphere" has also been used in the past (see Schrijver et al., 2003). "Astrosphere" has a longer history, with published usage in the literature dating at least back to 1978 (Fahr, 1978). The term "heliosphere" itself only dates back to the 1960s (Dessler, 1967).

The detection of astrospheric Ly $\alpha$ absorption represents an indirect detection of solar-like stellar winds, since astrospheres do not exist in the absence of a stellar wind. Furthermore, the amount of astrospheric absorption is dependent on the strength of the wind, so the astrospheric absorption has provided the first estimates of mass loss rates for solar-like stars. This article reviews the study of solar-like winds around other stars, especially results using the astrospheric Ly $\alpha$ absorption technique.

- In Section 2, some background material is provided about the solar wind, ISM, and heliosphere.

- In Section 3, techniques used to try to directly detect solar-like winds are reviewed.

- In Section 4, the astrospheric Ly $\alpha$ absorption diagnostic is described in detail. 
- Section 5 provides a review of what the astrospheric analyses have taught us about the solar wind, and discusses some implications of these results both within and outside the realm of solar/stellar physics.

- Finally, the article ends in Section 6 with some concluding comments about the future of this subject. 


\section{Background Material}

\subsection{The solar wind}

Before describing how solar-like winds are detected around other stars, it is worthwhile to briefly review what is known about the solar wind and how we study its properties. The solar wind was first detected through its role in the formation of aurorae and the creation of comet tails. As far back as 1896, Kristian Birkeland proposed that aurorae were due to particles emanating from the Sun (see review by Stern, 1989), while Biermann (1951) first described how "corpuscular radiation" from the Sun was responsible for the plasma tails of comets. Today almost everything we know about the solar wind comes from in situ measurements of its properties from satellites. These measurements date back to the Soviet Luna missions in 1959 (Gringauz et al., 1962) and NASA's Mariner 2 mission in 1962 (Neugebauer and Snyder, 1962). Numerous other spacecraft have participated in studying the solar wind since then, but of particular note are the venerable Voyager 1 and Voyager 2 satellites, which have returned data on the solar wind from 1977 through the present day (see Lazarus and McNutt Jr, 1990). More recently, the Ulysses spacecraft, launched in 1990 and still operating, has provided a first look at the solar wind outside of the ecliptic plane (McComas et al., 2000).

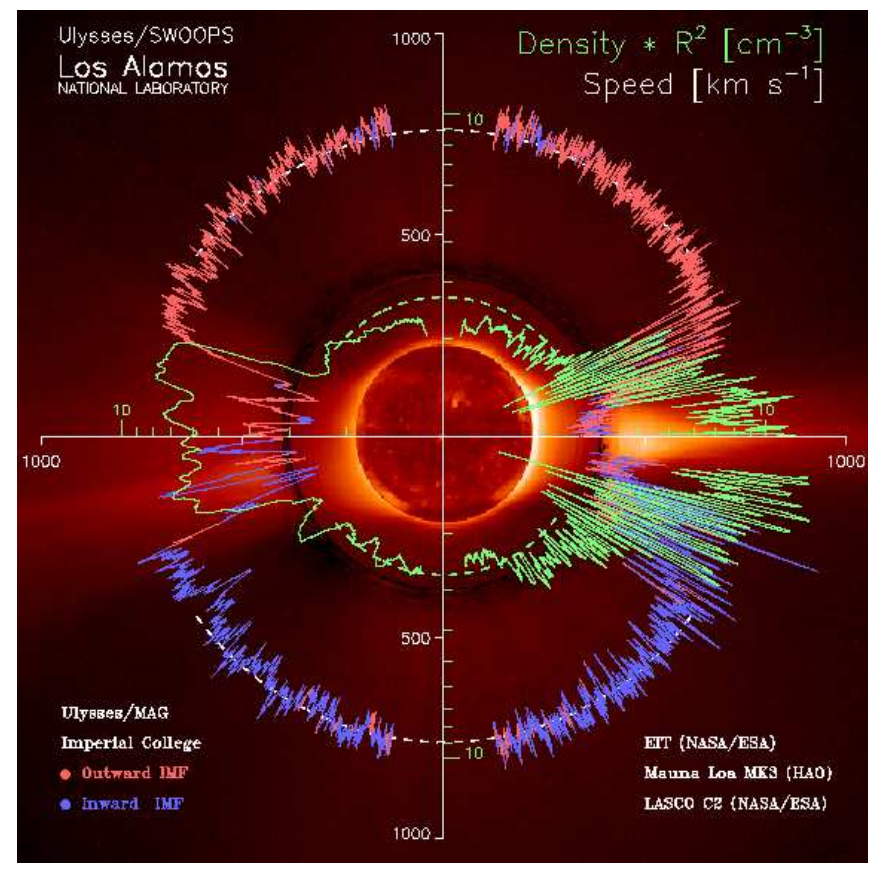

Figure 1: The solar wind velocity (red/blue line) and density (green line) observed by Ulysses as a function of ecliptic latitude (McComas et al., 2000). During solar minimum conditions, high latitudes are dominated by high speed, low density wind, while low latitudes see mostly lower speed wind with higher densities.

Within the ecliptic plane the solar wind is dominated by low speed streams with typical velocities, proton densities, and temperatures (at $1 \mathrm{AU}$ ) of $V=400 \mathrm{~km} \mathrm{~s}^{-1}, n\left(H^{+}\right)=5 \mathrm{~cm}^{-3}$, and $T=10^{5} \mathrm{~K}$, respectively, although high speed streams with lower densities and $V \approx 800 \mathrm{~km} \mathrm{~s}^{-1}$ are not uncommon (see Feldman et al., 1977). At the maximum of the 11-year solar activity cycle, similar solar wind behavior is seen at almost all latitudes. However, Figure 1 shows that 
at solar minimum the wind above $30^{\circ}$ ecliptic latitude is uniformly high speed, low density wind with $V \approx 800 \mathrm{~km} \mathrm{~s}^{-1}$ (McComas et al., 2000, 2002). This high speed wind originates from coronal holes, which are particularly prominent on the Sun during solar minimum conditions. These solar wind data imply a total mass loss rate for the Sun of $\dot{M}_{\odot} \approx 2 \times 10^{-14} M_{\odot} \mathrm{yr}^{-1}$. Although thermal temperatures for the wind at $1 \mathrm{AU}$ are of order $T=10^{5} \mathrm{~K}$, densities are low enough that the wind cannot equilibrate to this temperature, and the ionization state of the wind is actually frozen in at coronal temperatures closer to $T=10^{6} \mathrm{~K}$. Hydrogen is fully ionized, meaning that protons are the dominant constituents of the wind by mass.

Perhaps the most fundamental question to ask about the solar wind is why it exists. Addressing this question is also necessary to assess whether similar winds should exist around other stars. Even before satellites proved the existence of a more-or-less steady solar wind, Parker (1958) predicted that such a wind should be present due to the existence of the $10^{6} \mathrm{~K}$ corona surrounding the Sun. In Parker's model, the solar wind exists because of thermal expansion from the hot corona. The predictions of this simple model agree remarkably well with the observed properties of the low speed wind that dominates in the ecliptic plane, although additional wind acceleration mechanisms invoking MHD waves have been proposed to explain the high speed streams (see MacGregor and Charbonneau, 1994; Cranmer, 2002). Thus, any star that has a hot corona analogous to that of the Sun should also have a wind analogous to that of the Sun. Observations with X-ray satellites such as Einstein and ROSAT clearly demonstrate that coronae are a ubiquitous phenomenon among cool main sequence stars (see Schmitt, 1997; Hünsch et al., 1999), so solar-like winds should be present around all solar-like stars. However, that does not mean that they are easy to detect (see Section 1).

\subsection{The local interstellar medium}

The solar wind does not expand forever. Eventually it runs into the local interstellar medium (LISM). The interaction region between the solar wind and LISM is the subject of Section 2.3, and it is through analogous interaction regions around other stars that solar-like stellar winds can be detected (see Section 4), but before the wind/ISM interaction regions are discussed, it is necessary to review the properties of the undisturbed LISM.

The principle method by which one studies the ISM is by observing absorption lines that interstellar material produces in spectra of distant stars. These studies reveal that ISM column densities remain rather low within about $100 \mathrm{pc}$ of the Sun in most directions and then increase dramatically (Sfeir et al., 1999). This low density region is called the Local Bubble. Figure 2 shows a map of the Local Bubble in the Galactic plane (Lallement et al., 2003). The hot plasma within the Bubble has been detected directly from observations of the soft X-ray background (Snowden et al., 1995). Most locations within the Local Bubble are very hot $\left(T \sim 10^{6} \mathrm{~K}\right)$ and rarified $\left(n_{\mathrm{e}} \sim 10^{-3} \mathrm{~cm}^{-3}\right)$, and therefore completely ionized.

Although most of the volume of the Local Bubble consists of this hot material, the absorption line studies clearly demonstrate that there are cooler, partially neutral clouds embedded within the Local Bubble. Furthermore, since even the shortest lines of sight show absorption from H I and other low temperature species (Linsky, 1998; Linsky et al., 2000), the Sun must be located within one of these clouds. The cloud immediately surrounding the Sun has been called the Local Interstellar Cloud (LIC), which is roughly $5-7$ pc across and has a total mass of about $0.32 M_{\odot}$ (Redfield and Linsky, 2000). There are similar clouds that are apparently adjacent to the LIC (e.g. the "G" cloud and "Hyades" clouds, see Lallement and Bertin, 1992; Redfield and Linsky, 2001), although it is debatable whether the LIC is truly distinct from these clouds. Velocity gradients within a single cloud could in principle create the appearance of multiple clouds in absorption line studies.

The first evidence that the LIC is not entirely ionized came not from absorption line studies

Living Reviews in Solar Physics

http://www. livingreviews.org/lrsp-2004-2 


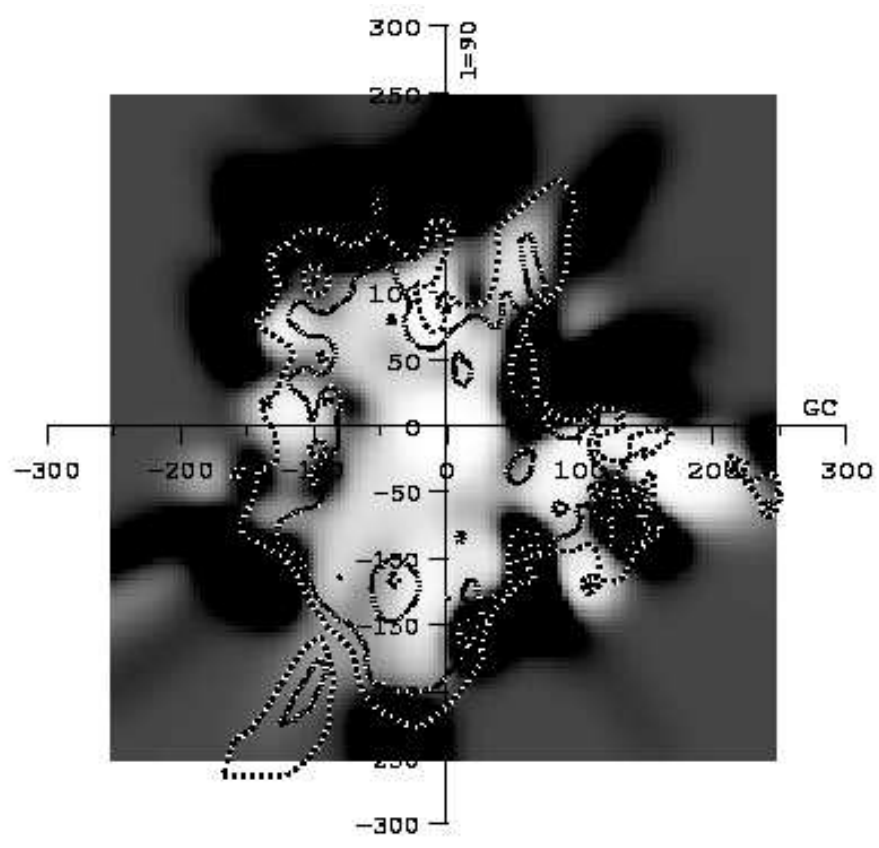

Figure 2: Map of the Local Bubble in the Galactic plane, where the contours indicate $20 m \AA$ and $50 \mathrm{~mA}$ equivalent widths for the Na I D2 line (Lallement et al., 2003). The distance scale is in parsecs.

but from observations of solar Ly $\alpha$ emission scattering off interstellar H I gas flowing into the heliosphere (Bertaux and Blamont, 1971; Quémerais et al., 1999, 2000). Interstellar atoms have also been observed directly with particle detectors on board spacecraft such as Ulysses (Witte et al., 1993, 1996). Both measurements of LIC material flowing through the heliosphere and LISM absorption line studies have been used to estimate the direction and magnitude of the LIC vector, and the resulting vectors are in good agreement. The heliocentric vector derived from absorption lines has a magnitude of $25.7 \mathrm{~km} \mathrm{~s}^{-1}$ directed towards Galactic coordinates $l=186.1^{\circ}$ and $b=-16.4^{\circ}$ (Lallement and Bertin, 1992; Lallement et al., 1995).

Other properties of the undisturbed LISM just beyond the heliosphere are less precisely known. Absorption line studies are hampered by probable variations of densities, temperatures, and ionization states within the LIC (see Cheng and Bruhweiler, 1990; Slavin and Frisch, 2002; Wood et al., 2003b), meaning that line-of-sight averages of these properties towards even the nearest stars are potentially different from the actual circumsolar LISM properties. Studies of LISM particles streaming through the heliosphere are hampered by the fact that the properties of these particles are often altered in the outer heliosphere, thereby requiring the assistance of models to extrapolate back to undisturbed LISM conditions (see Izmodenov et al., 2004). In any case, typical temperatures measured for the LIC are $T=6000-8000 \mathrm{~K}$, typical hydrogen densities are $n(\mathrm{HI})=0.1-0.2 \mathrm{~cm}^{-3}$, and typical proton and electron densities are $n\left(\mathrm{H}^{+}\right) \approx n_{\mathrm{e}}=0.04-0.2 \mathrm{~cm}^{-3}$ (Witte et al., 1993, 1996; Wood and Linsky, 1997, 1998; Izmodenov et al., 1999a; Redfield and Linsky, 2000; Frisch and Slavin, 2003). 


\subsection{The structure of the heliosphere}

Wind/ISM interactions provide the means by which solar-like stellar winds can be detected (see Section 4). Our understanding of these interactions relies heavily on a long history of efforts to model the solar wind/ISM interaction. This heliospheric modeling is summarized briefly here, but for more comprehensive reviews see Holzer (1989); Baranov (1990); Suess (1990) and Zank (1999).

Modeling the large scale structure of the heliosphere began not long after the solar wind's discovery (Parker, 1961, 1963). The basic structure of the heliosphere, which is shown schematically in Figure 3, is dominated by three prominent boundaries: the termination shock (TS), heliopause (HP), and bow shock (BS). The solar wind is highly supersonic, and the oval-shaped termination shock is where the radial wind is shocked to subsonic speeds. The $26 \mathrm{~km} \mathrm{~s}^{-1}$ laminar ISM flow is also generally believed to be supersonic, although in principle it could be subsonic if the poorly known ISM magnetic field is strong enough (Zank et al., 1996). Nevertheless, most heliospheric models assume the existence of a bow shock, where the ISM flow is shocked to subsonic speeds (see Figure 3). In between the TS and BS is the heliopause, which is a contact surface separating the plasma flows of the solar and interstellar winds.

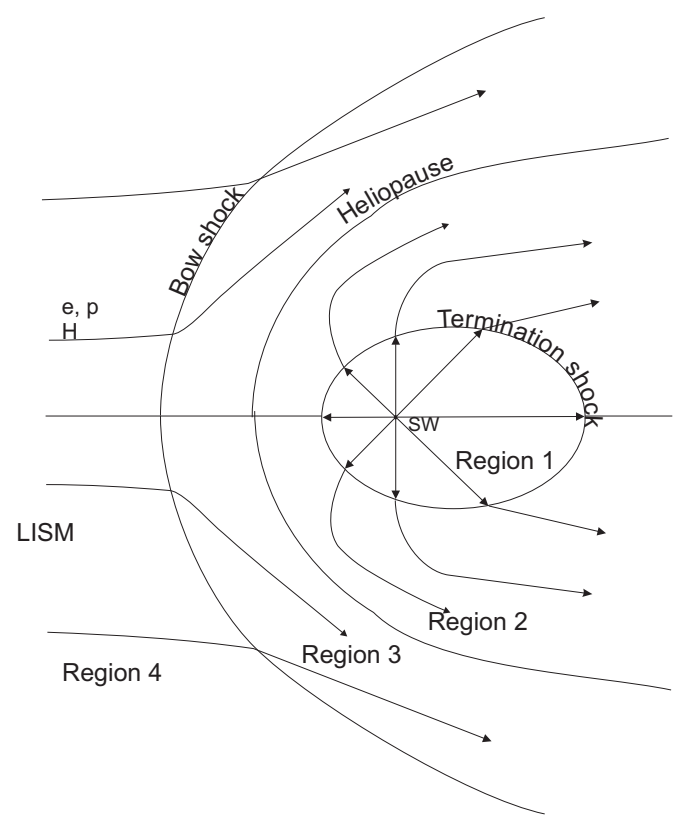

Figure 3: Schematic picture of the heliospheric interface from Izmodenov et al. (2002), which can be divided into the 4 regions shown in the figure, with significantly different plasma properties. Region 1: supersonic solar wind; Region 2: subsonic solar wind; Region 3: disturbed interstellar gas and plasma; and Region 4: undisturbed interstellar medium.

The heliospheric structure shown in Figure 3 is inferred almost entirely from hydrodynamic models. However, in 2004, Voyager 1 crossed the TS at a distance of 94 AU from the Sun in roughly the upwind direction of the ISM flow (Stone et al., 2005). Precursors of this crossing were seen years prior to the accepted crossing date (Krimigis et al., 2003; Burlaga et al., 2003; McDonald et al., 2003). At the time of this writing, Voyager 1's sister satellite Voyager 2 has begun to see these precursors but has not yet officially crossed the TS (Opher et al., 2006). The 94 AU TS distance measured by Voyager 1 is consistent with model predictions (Izmodenov et al., 2003). As for the HP and BS, recent models generally predict upwind distances of $\sim 140 \mathrm{AU}$ and

Living Reviews in Solar Physics

http://www. livingreviews.org/lrsp-2004-2 
$\sim 240$ AU, respectively. The Voyager satellites may not survive long enough to get out this far.

The plasma component of the LISM is diverted around the heliopause due to the strong plasma interactions, but neutrals in the LISM can penetrate into the solar system through the HP and TS. These neutrals were first detected through Ly $\alpha$ backscatter emission (Bertaux and Blamont, 1971). However, even after this discovery most hydrodynamic models of the heliosphere continued to ignore the neutrals since the collisional interactions involving neutrals are much weaker than those involving charged particles. Essentially, the assumption was made that the neutrals would pass through the heliosphere unimpeded, having little or no effect on the heliospheric structure.

It was recognized in the 1970s that the LISM neutrals could in fact play an important role in the solar wind/ISM collision through charge exchange interactions (Holzer, 1972; Wallis, 1975). However, trying to model this is very difficult, because the charge exchange sends the neutral $\mathrm{H}$ wildly out of thermal and ionization equilibrium. This means that simple fluid approximations break down and one has to resort to complex multi-fluid codes or ideally fully kinetic codes. It was not until much later that the first codes that treat the plasma and neutrals in a self-consistent manner were first developed (Baranov and Malama, 1993, 1995; Baranov and Zaitsev, 1995; Zank et al., 1996; Izmodenov et al., 1999a; Müller et al., 2000; Izmodenov et al., 2001). These models demonstrate that the heliospheric structure is indeed influenced significantly by the neutrals in many different ways.

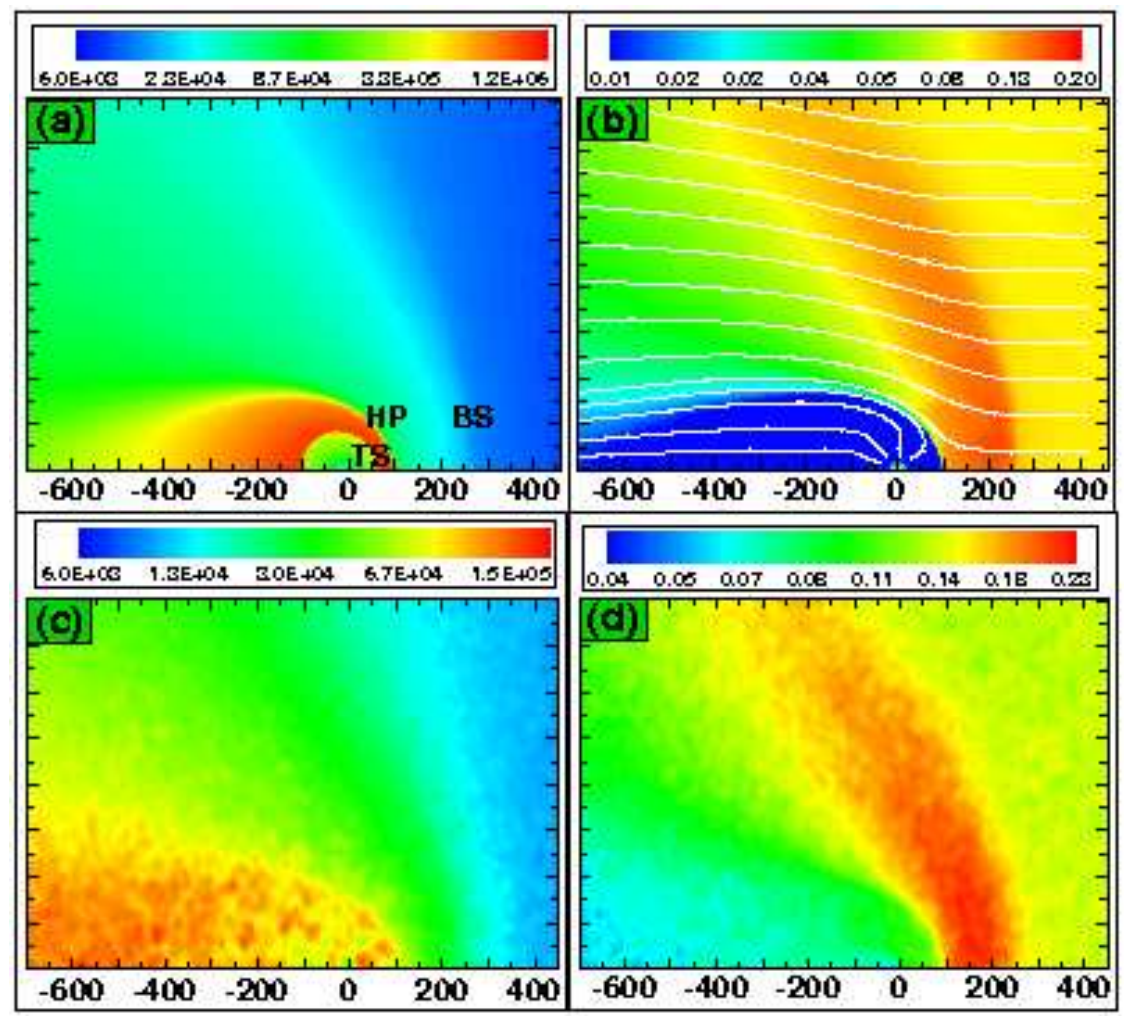

Figure 4: (a) Proton temperature, (b) proton density, (c) neutral hydrogen temperature, and (d) neutral hydrogen density distributions for a heliospheric model from Wood et al. (2000b). The positions of the termination shock (TS), heliopause (HP), and bow shock (BS) are indicated in (a), and streamlines indicating the plasma flow direction are shown in (b). The distance scale is in $A U$.

For purposes of this article, the importance of the development of heliospheric codes that treat 
neutrals properly is that it is only because of the existence of neutral hydrogen in the LISM that heliospheric and astrospheric Ly $\alpha$ absorption is detectable, and it is only because of the existence of the self-consistent codes developed to model neutrals in the heliosphere that we can model the astrospheric absorption and extract stellar mass loss rates from the data. Figure 4 shows a heliospheric model that uses a hybrid kinetic code, where the protons are modeled as a fluid but a kinetic code is used for the neutrals (Lipatov et al., 1998; Müller et al., 2000; Wood et al., 2000b). The strong plasma interactions heat and compress LISM protons in between the HP and BS, and thanks to charge exchange processes these high temperatures and densities are transmitted to the neutral H. As a consequence, the heliosphere and astrospheres are permeated by a population of hot hydrogen, which produces a substantial amount of Ly $\alpha$ absorption in HST observations of nearby stars. Most of this absorption comes from the "hydrogen wall" region in between the HP and BS, where densities of the hot H I are particularly high (see Figure 4d). The heliospheric and astrospheric Ly $\alpha$ diagnostic is described in detail in Section 4. 


\section{Direct Wind Detection Techniques}

The astrospheric Ly $\alpha$ absorption diagnostic described in detail in Section 4 represents only an indirect detection of stellar winds, since the H I that produces the absorption is essentially LISM rather than wind material. The H I is nevertheless heated by its interaction with the stellar wind, and the astrospheric absorption has therefore proven to be very useful for successfully detecting and measuring solar-like winds around other stars. However, there have been attempts to detect these winds more directly, and these mostly unsuccessful efforts are briefly summarized here.

One can try to look for free-free radio emission from solar-like winds, since they are fully ionized and should therefore produce emission at some level. However, current radio telescopes can only detect winds if they are much stronger than that of the Sun. There have been some claims of very high mass loss rates for a few very active stars using observations at millimeter wavelengths (Mullan et al., 1992), but these interpretations of the data are highly controversial (Lim and White, 1996; van den Oord and Doyle, 1997). The problem is that the coronae of these active stars are also sources of radio emission, which makes problematic the identification of a wind as the source of the emission. Furthermore, it has been argued that massive winds around active stars should absorb the flaring coronal emission that is often observed from these stars, suggesting that massive winds cannot be present (Lim and White, 1996). Nondetections of radio emission have been used to derive upper limits to the mass loss rates of various stars, but these upper limits are typically $2-3$ orders of magnitude higher than the solar mass loss rate, so these are not very stringent constraints (Brown et al., 1990; Drake et al., 1993; Lim et al., 1996b; Gaidos et al., 2000).

Variable ultraviolet absorption features observed from the close, eclipsing binary V471 Tau (K2 V+DA) have been interpreted as being due to a wind from the K2 V star (Mullan et al., 1989; Bond et al., 2001). Even if this interpretation is correct, it is questionable whether the wind produced by this star can be considered to be truly "solar-like" given the close presence of the white dwarf companion. In addition, instead of a spherically symmetric wind it has been proposed that the UV absorption could instead be indicative of coronal material being funneled directly from the $\mathrm{K} 2 \mathrm{~V}$ star to the white dwarf through the magnetospheric interaction of the two stars (Lim et al., 1996a).

One final wind detection technique that has been proposed is to look for X-ray emission surrounding nearby stars, caused by charge exchange between highly ionized heavy atoms in the stellar wind and inflowing LISM neutrals. This is very analogous to the process by which comets produce X-rays (see Lisse et al., 2001; Cravens, 2002). In the heliosphere, this charge exchange X-ray emission may be responsible for a significant fraction of the observed soft X-ray background (Cravens, 2000). Wargelin and Drake (2002) searched for circumstellar X-ray emission in Chandra observations of the nearest star, Proxima Cen, but they failed to detect any. Based on this nondetection, they quote an upper limit for Proxima Cen's mass loss rate of $\dot{M}<14 \dot{M}_{\odot}$. This can be compared with the upper limit of $\dot{M}<350 \dot{M}_{\odot}$ derived from a nondetection of radio emission from Proxima Cen (Lim et al., 1996b), and the upper limit of $\dot{M}<0.2 \dot{M}_{\odot}$ derived from the nondetection of astrospheric Ly $\alpha$ absorption (Wood et al., 2001). The astrospheric Ly $\alpha$ absorption diagnostic (see Section 4) is roughly two orders of magnitude more sensitive than the X-ray diagnostic and roughly three orders of magnitude more sensitive than the radio measurement. 


\section{Detecting Winds Through Astrospheric Absorption}

\subsection{Analyzing H I Lyman-alpha lines}

Spectroscopic analyses of stellar H I Lyman- $\alpha$ lines have proven to be the best way so far to clearly detect and measure weak solar-like winds, but analysis of this line is complex. The history of Ly $\alpha$ absorption observations and analyses is summarized here, with emphasis on how these studies eventually led to the detection of solar-like stellar winds.

The Ly $\alpha$ line at $1216 \AA$ is the most fundamental transition of the most abundant atom in the universe. As such, the line is a valuable diagnostic for many purposes. For cool stars, the Ly $\alpha$ line is a strong radiative coolant for stellar chromospheres, and is therefore an important chromospheric diagnostic. Cool stars produce very little continuum emission at $1216 \AA$, so Ly $\alpha$ lines observed from cool stars are strong, isolated emission lines. However, these lines are always heavily contaminated by interstellar absorption.

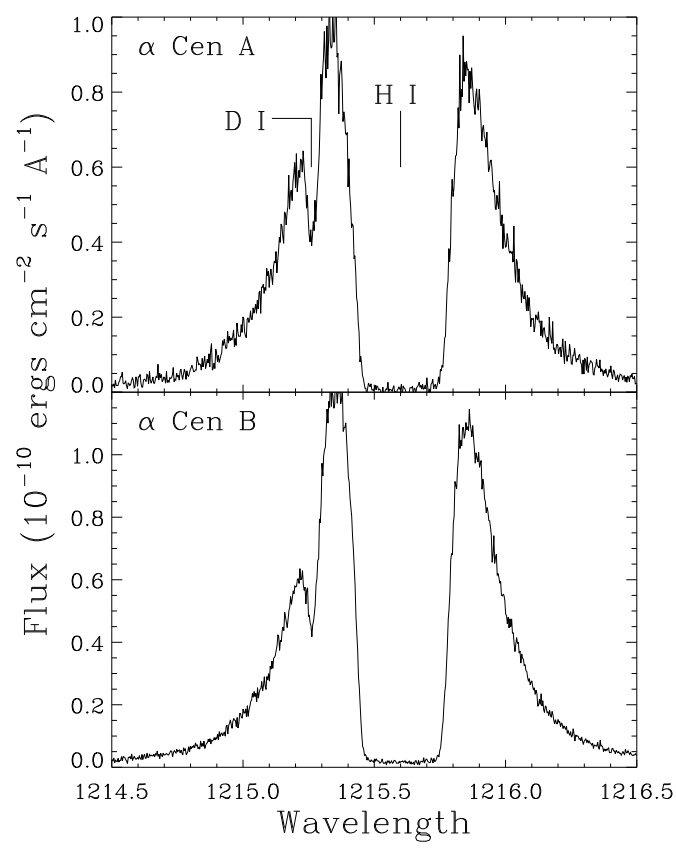

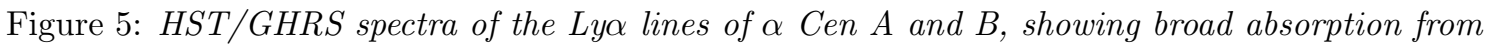
interstellar H I and narrow absorption from D I (Linsky and Wood, 1996).

Figure 5 shows the Ly $\alpha$ lines observed from the two members of the $\alpha$ Cen binary, which is the nearest star system to us at a distance of only $1.3 \mathrm{pc}$. The centers of the stellar emission lines are obliterated by broad, saturated H I absorption. Narrower absorption from deuterium (D I) is visible $-0.33 \AA$ from the $\mathrm{H}$ I absorption. The D I absorption is entirely from interstellar material in between $\alpha$ Cen and the Sun, and interstellar absorption also accounts for much of the H I absorption. The H I and D I absorption lines are therefore valuable diagnostics for the LISM, and stellar Ly $\alpha$ data like that in Figure 5 have proven to be useful for measuring the properties of warm, neutral ISM gas, and also for mapping out the distribution of this gas in the vicinity of the Sun (see Linsky et al., 2000; Redfield and Linsky, 2000). 
The importance of these data is magnified further by the measurements the data provide of $\mathrm{D} / \mathrm{H}$ ratios. The LISM D/H ratio has important applications for both cosmology and our understanding of Galactic chemical evolution (see McCullough, 1992; Linsky, 1998; Moos et al., 2002; Wood et al., 2004). The light element abundances in the universe are powerful diagnostics for Big Bang nucleosynthesis calculations, with the exact abundances depending on the cosmic baryon density, $\Omega_{\mathrm{b}}$. The deuterium abundance is particularly sensitive to $\Omega_{\mathrm{b}}$, so measuring the primordial $\mathrm{D} / \mathrm{H}$ ratio and thereby constraining cosmological models has been a major goal in astronomy (see Boesgaard and Steigman, 1985; Burles et al., 2001). The most accurate meaningful D/H measurements come from analyses of LISM absorption like that in Figure 5. Unfortunately, the LISM D/H ratio only provides a lower limit to the primordial $\mathrm{D} / \mathrm{H}$ ratio. Since deuterium is destroyed in stellar interiors, the $\mathrm{D} / \mathrm{H}$ ratio is expected to have decreased with time, so Galactic chemical evolution models are required to extrapolate back to a primordial value (see Prantzos, 1996; Tosi et al., 1998; Chiappini et al., 2002). Primordial D/H values can also be measured more directly by measuring $\mathrm{D} / \mathrm{H}$ in more pristine intergalactic material (see Kirkman et al., 2003).

The desire to improve our understanding of the LISM and measure D/H has provided the primary impetus behind stellar Ly $\alpha$ analyses. This work dates back to Copernicus (York and Rogerson, 1976; Dupree et al., 1977), which was the first ultraviolet astronomical satellite to provide high quality stellar Ly $\alpha$ spectra. Copernicus was followed by the long-lived International Ultraviolet Explorer (IUE) satellite, which also provided Ly $\alpha$ spectra that could be analyzed for LISM and D/H purposes (see Murthy et al., 1987; Diplas and Savage, 1994). However, it was the Goddard High Resolution Spectrograph (GHRS) instrument aboard HST that was the first UV spectrometer capable of fully resolving the D I and H I absorption line profiles. The GHRS spectrometer was replaced by the Space Telescope Imaging Spectrometer (STIS) in 1997.

The first Ly $\alpha$ analyses from HST data were for the lines of sight to Capella and Procyon (Linsky et al., 1993, 1995). However, the third analysis, which was of the $\alpha$ Cen data shown in Figure 5, presented a dilemma. The observed H I absorption is simply inconsistent with D I and other ISM absorption lines (Mg II, Fe II, etc.). The D I absorption and the other non-H I lines are centered at a heliocentric velocity of $v=-18.0 \pm 0.2 \mathrm{~km} \mathrm{~s}^{-1}$, and the widths of these lines suggest an interstellar temperature of $T=5400 \pm 500 \mathrm{~K}$. However, the H I absorption implies $v=-15.8 \pm 0.2 \mathrm{~km} \mathrm{~s}^{-1}$ and $T=8350 \mathrm{~K}$. In other words, the $\mathrm{H} \mathrm{I}$ absorption is broader than it should be, and it is also redshifted by $2.2 \mathrm{~km} \mathrm{~s}^{-1}$ from where it should be. (Linsky and Wood, 1996). Interestingly enough, the H I redshift was also discerned earlier in much lower quality IUE spectra (Landsman et al., 1984).

Linsky and Wood (1996) interpreted the problem as being due to the presence of an extra H I absorption component that contributes no absorption to any of the weaker ISM lines. This was also one of the hypotheses suggested by Landsman et al. (1984) based on the IUE data. Two-component fits to the HST/GHRS data from Linsky and Wood (1996) suggest that the extra H I absorption can be explained by the existence of a redshifted $\mathrm{H}$ I absorption component with a temperature of $T \approx 30000 \mathrm{~K}$ and a column density $\left(\right.$ in $\mathrm{cm}^{-2}$ ) of $\log N(\mathrm{HI}) \approx 15.0$. This temperature is much hotter than typical LISM material, and the column density is almost three orders of magnitude lower than the ISM H I column density towards $\alpha$ Cen. The low column density would explain why the absorption component is only seen in the H I line and not in any of the other ISM lines from atomic species with much lower abundances. However, the interpretation for this absorption component was initially a mystery.

Fortuitously, the $\alpha$ Cen Ly $\alpha$ analysis was being performed at about the same time as the first heliospheric models including neutrals in a self-consistent manner were being developed (see Section 2.3). One session of the 1995 IUGG (International Union of Geodesy and Geophysics) General Assembly brought together interstellar and heliospheric experts, and it was realized during that meeting that the heated heliospheric hydrogen predicted by the new heliospheric models had precisely the right properties to account for the extra $\alpha$ Cen absorption component. It was quickly 
realized that if hot hydrogen existed around the Sun, then it should exist around other solar-like stars as well, so the initial $\alpha$ Cen analysis suggested that the excess H I absorption could be partly due to astrospheric as well as heliospheric absorption (Linsky and Wood, 1996). Thus was born a new way to detect and study stellar winds.

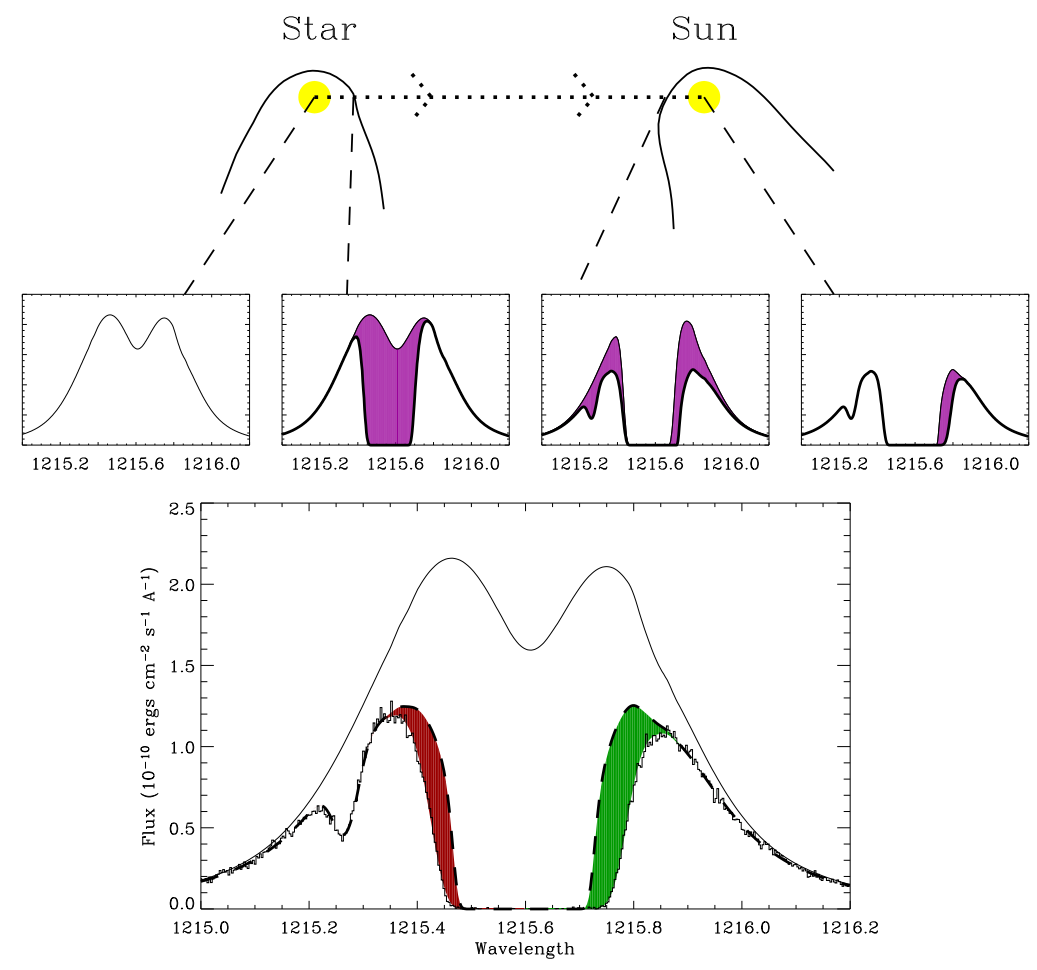

Figure 6: Schematic diagram showing how a stellar Lya profile changes from its initial appearance at the star and then through various regions that absorb parts of the profile before it reaches an observer at Earth: the stellar astrosphere, the LISM, and finally the heliosphere (Wood et al., 2003b). The lower panel shows the actual observed Ly $\alpha$ profile of $\alpha$ Cen B. The upper solid line is the assumed stellar emission profile and the dashed line is the ISM absorption alone. The excess absorption is due to heliospheric H I (green shading) and astrospheric H I (red shading).

More work was required to verify this interpretation of the Ly $\alpha$ data. Gayley et al. (1997) made the first direct comparison between the $\alpha$ Cen Ly $\alpha$ data and the predictions of various heliospheric models. This work demonstrated that heliospheric Ly $\alpha$ absorption can only account for the excess absorption seen on the red side of the $\operatorname{Ly} \alpha$ line, and that astrospheric absorption is required to explain the excess absorption seen on the blue side of the line. A more refined interpretation for the $\alpha$ Cen Ly $\alpha$ absorption was therefore developed, and is shown schematically in Figure 6 . The four middle panels in Figure 6 show what happens to the $\alpha$ Cen B Ly $\alpha$ line profile as it journeys from the star towards the Sun. The Ly $\alpha$ profile first has to traverse the hot hydrogen in the star's astrosphere, which erases the central part of the line. The Ly $\alpha$ emission then makes the 
long interstellar journey, resulting in additional absorption, including some from D I. Finally, the profile has to travel through the heliosphere, resulting in additional absorption on the red side of the line. Most of the astrospheric and heliospheric absorption is from material in the "hydrogen wall" region mentioned at the end of Section 2.3 (see Figure 4).

Why is the heliospheric absorption redshifted relative to the interstellar absorption? For the $\alpha$ Cen line of sight, which is roughly in the upwind direction relative to the LISM flow seen by the Sun, the heliospheric absorption is redshifted primarily because of the deceleration and deflection of interstellar material as it crosses the bow shock. Heliospheric models predict that heliospheric Ly $\alpha$ absorption should always be redshifted relative to the ISM absorption, even in downwind directions, although the physical explanation for the redshift in the downwind direction is more complicated (Izmodenov et al., 1999b; Wood et al., 2000b). Conversely, astrospheric absorption will always be blueshifted relative to the ISM absorption, since we are viewing that absorption from outside the astrosphere rather than inside. It is very fortunate that heliospheric and astrospheric material produce excess absorption on opposite sides of the Ly $\alpha$ line, as this makes it possible to identify the source of the absorption.

The bottom panel of Figure 6 shows the observed Ly $\alpha$ profile of $\alpha$ Cen B (Linsky and Wood, 1996). As mentioned above, the non-H I ISM lines observed towards $\alpha$ Cen suggest $v=-18.0 \pm$ $0.2 \mathrm{~km} \mathrm{~s}^{-1}$ and $T=5400 \pm 500 \mathrm{~K}$ for the ISM material in this direction. The dashed line in the bottom panel of Figure 6 shows what the H I absorption looks like when forced to be consistent with these results. No matter what is assumed for the stellar line profile, and no matter what is assumed for the ISM H I column density, there is always excess absorption on both sides of the H I absorption feature that cannot be explained by ISM absorption. As suggested above, the red side excess is best interpreted as heliospheric absorption and the blue side excess is best interpreted as astrospheric absorption.

This example illustrates how heliospheric and astrospheric absorption is detected. The ISM H I absorption is estimated by forcing the $\mathrm{H}$ I fit parameters to be consistent with D I and other ISM lines. In many cases, this still leads to excellent fits to the data, but in some cases there is evidence for excess H I absorption on one or both sides of the line, indicating the presence of heliospheric and/or astrospheric absorption. The Ly $\alpha$ analyses can be simplified even further if one assumes that $\mathrm{D} / \mathrm{H}=1.5 \times 10^{-5}$, in addition to forcing $v$ and $T$ to be consistent for $\mathrm{D} \mathrm{I}$ and $\mathrm{H}$ I. This assumption should be valid for nearby stars, since recent work suggests that $\mathrm{D} / \mathrm{H} \approx 1.5 \times 10^{-5}$ throughout the Local Bubble, with no evidence for variation (Linsky, 1998; Moos et al., 2002; Wood et al., 2004).

The heliospheric/astrospheric interpretation of the excess Ly $\alpha$ absorption has strong theoretical support, but additional evidence for the validity of this interpretation is still valuable. The best purely empirical demonstration that it is correct comes by comparing the Ly $\alpha$ absorption observed towards $\alpha$ Cen with that observed towards a distant $\mathrm{M}$ dwarf companion of the $\alpha$ Cen system called Proxima Cen (Wood et al., 2001). This comparison is made in Figure 7. The Ly $\alpha$ absorption profiles agree well on the red side of the line where the heliospheric absorption is located. However, the blue-side excess absorption seen towards $\alpha$ Cen is not seen towards Proxima Cen. This means that the blue-side excess absorption seen towards $\alpha$ Cen has to be from circumstellar material surrounding $\alpha$ Cen that does not extend as far as the distant companion Proxima Cen $(\sim 12000 \mathrm{AU}$ away), consistent with the astrospheric interpretation. Apparently, Proxima Cen must have a weaker wind than the $\alpha$ Cen binary, which results in a much smaller astrosphere and much less astrospheric Ly $\alpha$ absorption. (The two $\alpha$ Cen stars are close enough that they will share the same astrosphere, and the astrospheric absorption will therefore be characteristic of the combined winds of both stars.) This example suggests how the astrospheric absorption might be used as a diagnostic for the mass loss rates of solar-like stars, which is a subject that is discussed in detail in Section 4.3. 


\subsection{Comparing heliospheric absorption with model predictions}

In this article, we are more interested in the astrospheric Ly $\alpha$ absorption than the heliospheric absorption, since we are concerned with using the astrospheric detections to measure the properties of solar-like stellar winds. However, hydrodynamic models of the astrospheres are required to do this (see Section 4.3). In order to believe the results of these models, it is crucial to demonstrate that they are properly extrapolated from heliospheric models that can reproduce observed heliospheric absorption. Thus, the heliospheric absorption and efforts to reproduce it using models are reviewed in this section.

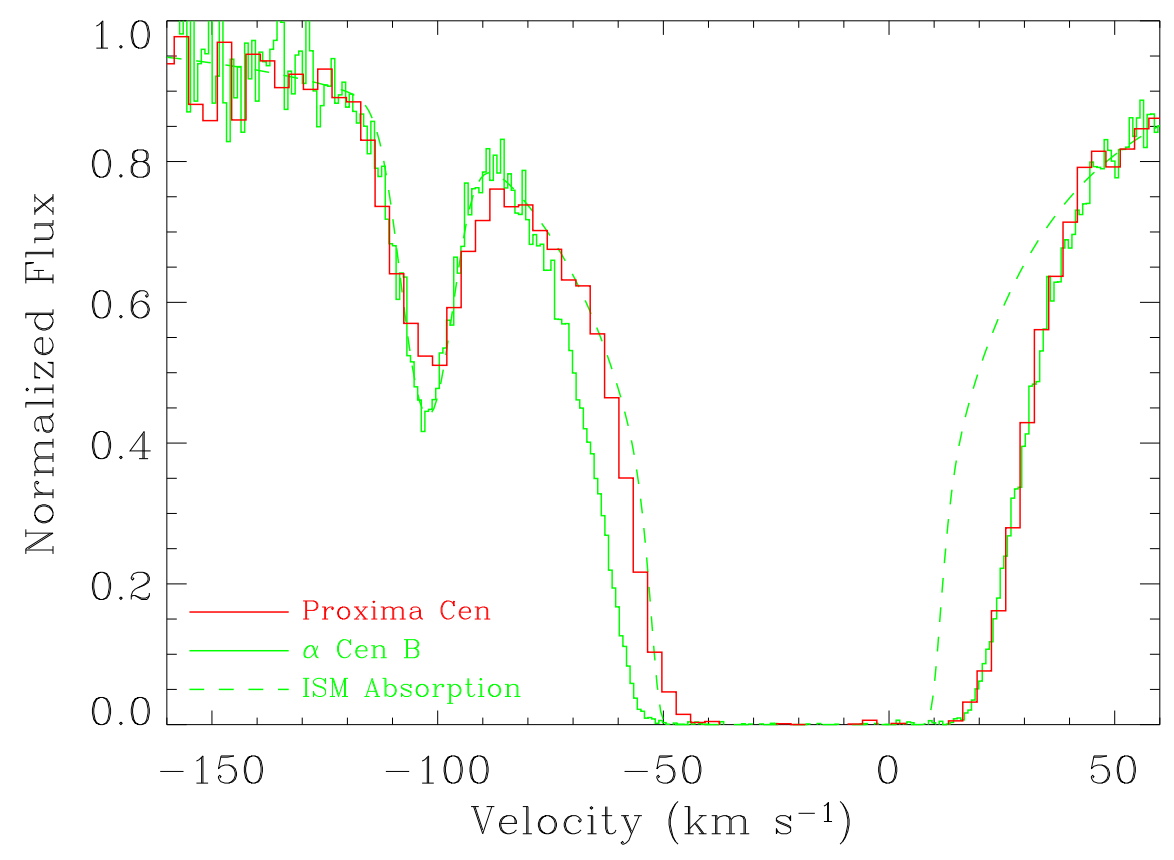

Figure 7: Comparison between the Lya spectra of $\alpha$ Cen B (green histogram) and Proxima Cen (red histogram) from Wood et al. (2001). The inferred ISM absorption is shown as a green dashed line. The Alpha/Proxima Cen data agree well on the red side of the $H I$ absorption, but on the blue side the Proxima Cen data do not show the excess Lya absorption seen toward $\alpha$ Cen (i.e. the astrospheric absorption).

Gayley et al. (1997) were the first to clearly demonstrate that heliospheric models are capable of reproducing the observed excess Ly $\alpha$ absorption, at least on the red side of the line (see Section 4.1). However, the exact amount of absorption predicted depends on exactly what properties are assumed for the surrounding LISM. Since many of these LISM properties are not precisely known (see Section 2.2), there is the hope that the heliospheric absorption can be used as a diagnostic for the LISM properties, such that the heliospheric absorption is only reproduced when the correct LISM parameters are assumed. In practice, this has proven to be very difficult.

One problem has been finding enough detections of heliospheric Ly $\alpha$ absorption to provide proper constraints for the models, although the situation has been gradually improving. The first heliospheric absorption detection was for the $\alpha$ Cen line of sight described in detail in Section 4.1. The second detection was for the downwind line of sight towards Sirius (Izmodenov et al., 1999b),

Living Reviews in Solar Physics

http://www. livingreviews . org/lrsp-2004-2 
though the analysis of Hébrard et al. (1999) suggests that this detection is not very secure. The upwind line of sight towards 36 Oph (Wood et al., 2000a) provided the third detection, and there is a marginal detection for the crosswind line of sight towards HZ 43 (Kruk et al., 2002). An HST archival Ly $\alpha$ survey by Wood et al. (2005b) resulted in four more detections (70 Oph, $\xi$ Boo, 61 Vir, and HD 165185). Most recently, a detailed analysis of all reconstructed stellar Ly $\alpha$ lines based on HST data has found evidence for very broad, weak heliospheric absorption for three lines of sight ( $\chi^{1}$ Ori, HD 28205, HD 28568) observed within $20^{\circ}$ of the downwind direction (Wood et al., 2007b). This brings the grand total of absorption detections to 11. In addition, Lemoine et al. (2002) and Vidal-Madjar and Ferlet (2002) have claimed to find evidence for weak heliospheric absorption towards the similar Capella and G191-B2B lines of sight, but these claims rely on subtle statistical arguments rather than clearly visible excess absorption.

The heliospheric absorption detections can be supplemented with other lines of sight observed by HST that at least provide useful upper limits for the amount of heliospheric absorption. Figure 8 shows the Ly $\alpha$ absorption profiles observed for three of the lines of sight with detected heliospheric

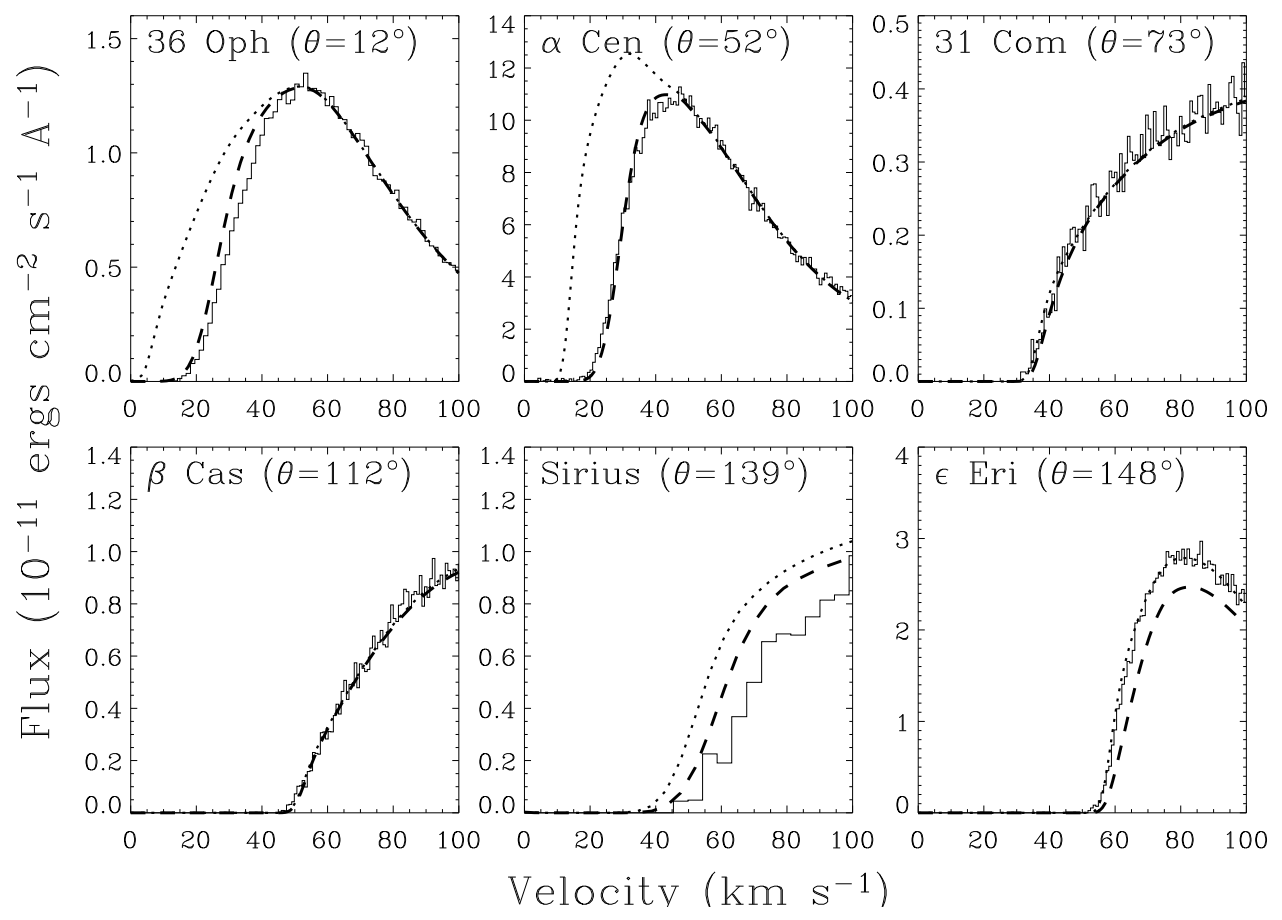

Figure 8: Comparison of the H I absorption predicted by a four-fluid heliospheric model (dashed lines) and the observations, where the model heliospheric absorption is shown after having been added to the ISM absorption (dotted lines). Reasonably good agreement is observed, although there is a slight underprediction of absorption towards 36 Oph and Sirius, and a slight overprediction towards $\epsilon$ Eri (Wood et al., 2000b).

absorption (36 Oph, $\alpha$ Cen, and Sirius) and three additional lines of sight with nondetections (31 Com, $\beta$ Cas, and $\epsilon$ Eri). The figure zooms in on the red sides of the profiles where the heliospheric absorption should be located. The $\theta$ angles in the figure indicate the angle of the line of sight with respect to the upwind direction of the interstellar flow. The six sight lines sample diverse orientiation angles, ranging from the nearly upwind direction towards $36 \mathrm{Oph}\left(\theta=12^{\circ}\right)$ to the nearly downwind line of sight to $\epsilon$ Eri $\left(\theta=148^{\circ}\right)$. The dotted lines show the ISM absorption 
alone, constrained by forcing consistency with D I (see Section 4.1) - only 36 Oph, $\alpha$ Cen, and Sirius show excesses that reveal the presence of heliospheric absorption. A successful heliospheric model should accurately reproduce the amount of heliospheric absorption detected towards these three stars, while predicting no detectable absorption towards the other three.

Figure 8 shows a model that agrees reasonably well with the data, despite a slight overprediction of absorption towards $\epsilon$ Eri and slight underpredictions for $36 \mathrm{Oph}$ and Sirius (Wood et al., $2000 \mathrm{~b})$. This model assumes $T=8000 \mathrm{~K}, n(\mathrm{HI})=0.14 \mathrm{~cm}^{-3}$, and $n\left(\mathrm{H}^{+}\right)=0.10 \mathrm{~cm}^{-3}$ for the ambient LISM, parameters well within the range of values inferred by other means (see Section 2.2). However, not all heliospheric models that assume these parameters find agreement with the data.

This brings us to the second problem with trying to infer ambient LISM parameters from the heliospheric Ly $\alpha$ data: Results currently seem to be very model dependent. It is mentioned in Section 2.3 just how difficult it is to properly consider neutrals in heliospheric models due to charge exchange processes driving the neutral $\mathrm{H}$ out of thermal equilibrium. The model used in Figure 8 is a "four-fluid" model of the type developed by Zank et al. (1996), where one fluid represents the protons, and three distinct fluids are used to represent the neutral hydrogen, one fluid for each distinct region where charge exchange occurs (inside the TS, between the TS and HP, and between the HP and BS). However, there are other approaches, such as the hybrid kinetic code of Müller et al. (2000) and the Monte Carlo kinetic code of Baranov and Malama (1993, 1995). The heliospheric absorption predicted by these kinetic models is not identical to that predicted by the four-fluid models (Wood et al., 2000b; Izmodenov et al., 2002). Currently the kinetic models seem to have more difficulty reproducing the observed heliospheric absorption than the four-fluid models, especially in downwind directions where they tend to predict too much absorption. However, the kinetic models should in principle yield more accurate velocity distributions for the neutral $\mathrm{H}$ than codes with multi-fluid approximations. A complex multi-component treatment of the protons in the heliosphere seems to improve the kinetic models' ability to fit the data (Malama et al., 2006; Wood et al., 2007b). Clearly more work is required to attain some sort of convergence in the models before LISM parameters can be unambiguously derived from the data.

However, a third difficulty with using the heliospheric absorption to infer ambient LISM properties is that the absorption may not be as sensitive to these properties as one might wish. Izmodenov et al. (2002) experiment with different LISM proton and neutral hydrogen densities and find surprisingly little change in the predicted Ly $\alpha$ absorption, at least in upwind and sidewind directions. This may be bad news for the diagnostic power of the heliospheric absorption, but it is actually good news for the astrospheric analyses that are described in Section 4.3. In using astrospheric models to help extract stellar mass loss rates from the astrospheric absorption, one has to assume that the LISM does not vary much from one location to another. The results of Izmodenov et al. (2002) suggest that the models are not very sensitive to the modest variations in LISM properties that one might expect to be present in the solar neighborhood.

Finally, there are some aspects of heliospheric physics that are only beginning to be considered in the models. The models mentioned previously do not consider either the heliospheric magnetic field carried outwards by the solar wind (see Nerney et al., 1991), or the poorly known interstellar magnetic field. Not only is a proper MHD treatment of the heliosphere difficult, but the problem is inherently three dimensional, whereas the models mentioned previously assume a $2 \mathrm{D}$ axisymmetric geometry. Using a 2D approach, Florinski et al. (2004) find that a strong ISM field oriented parallel to the LISM flow does not yield significantly different predictions for heliospheric Ly $\alpha$ absorption than models without magnetic fields. However, 3D models are required to include the heliospheric field, and 3D models are also required to consider ISM field orientations other than parallel to the flow vector.

Initial 3D models developed to model these effects (see Linde et al., 1998) did not include neutrals in a self-consistent manner. Dealing with both neutrals and magnetic fields properly in a $3 \mathrm{D}$ model is a very formidable problem. Nevertheless, the $3 \mathrm{D}$ models without neutrals do

Living Reviews in Solar Physics

http://www. livingreviews.org/lrsp-2004-2 
suggest that MHD effects could in principle lead to changes in the heliospheric structure that could affect the Ly $\alpha$ absorption. Examples include the unstable jet sheet and north-south asymmetries predicted by Opher et al. (2003, 2004, 2006). In addition, Ratkiewicz et al. (1998) find that if the LISM magnetic field is skewed with respect to the ISM flow, the effective nose of the heliosphere could be significantly shifted from the upwind direction. Even in the absence of magnetic fields, latitudinal variations in solar wind properties could also cause asymmetries in the heliosphere (Pauls and Zank, 1997). It is possible that all these asymmetries suggested by 3D models could be detectable in Ly $\alpha$ absorption. However, neutrals must be included properly in the models to make clearer predictions. Only very recently has this been done (Izmodenov et al., 2005; Pogorelov et al., 2006). Wood et al. (2007a) have made the first comparison between such models and the Ly $\alpha$ data, finding that the absorption predicted by the models is modestly affected by the assumed LISM field strength and orientation, allowing some constraints on these quantities to be inferred from the data. However, with the absorption being modestly dependent on so many uncertain LISM properties, including particle densities, it is probably unreasonable to expect analysis of the absorption by itself to yield a single set of acceptable LISM parameters.

\subsection{Measuring stellar mass loss rates}

The $\alpha$ Cen line of sight provided the first detection of astrospheric Ly $\alpha$ absorption (Linsky and Wood, 1996), but the use of models by Gayley et al. (1997) was necessary to clearly demonstrate that heliospheric absorption could not explain the blue-side excess as well as the stronger redside excess (see Figure 6). By that time, there were already two other lines of sight, $\epsilon$ Ind and $\lambda$ And, with excess Ly $\alpha$ absorption found only on the blue side of the absorption line, which clearly could not be heliospheric in origin (Wood et al., 1996). This excess absorption was immediately interpreted as being solely astrospheric. Thus, a case could be made for either $\alpha$ Cen AB or $\epsilon$ Ind $/ \lambda$ And being the first stars with detected solar-like coronal winds.

There are now a total of 13 published detections of astrospheric absorption. These detections are listed in Table 1, in addition to Proxima Cen since the nondetection for Proxima Cen is used to derive an upper limit for its mass loss rate (see below).

Some of these stars are binaries where the individual components are clearly close enough that both stars will reside within the same astrosphere, meaning that the observed astrospheric absorption is indicative of the combined mass loss of both stars. For these binaries ( $\alpha$ Cen, 36 Oph, and $\lambda$ And), the spectral types of both stars are listed in Table 1 and the listed stellar surface areas are the combined areas of both stars. In contrast, $61 \mathrm{Cyg}$ A's companion is far enough away that 61 Cyg A should have an astrosphere all to itself, so it is listed alone in Table 1.

Three detections are flagged as uncertain in Table 1 for various reasons that will not be discussed here (see Wood et al., 2002, 2005b). Results regarding these three lines of sight should be considered with caution. There was originally another uncertain astrospheric detection, 40 Eri A (Wood and Linsky, 1998; Wood et al., 2002), which has now been dropped entirely from the list of astrospheric detections. Since astrospheric hydrogen scatters stellar Ly $\alpha$ photons, solar-like stars should in principle be surrounded by faint nebulae of astrospheric Ly $\alpha$ emission. There was an unsuccessful attempt to detect this emission surrounding 40 Eri A, and the lack of success was used to argue that the tentative detection of astrospheric absorption for 40 Eri A could not be correct (Wood et al., 2003a).

The astrospheric absorption detections represent an indirect detection of solar-like winds from the observed stars, and the amount of absorption observed has diagnostic power. Frisch (1993) envisioned using astrospheres as probes for the interstellar medium, but so far work has focused on the use of the astrospheric absorption for estimating stellar mass loss rates. The higher the stellar mass loss rate, the larger the astrosphere will be, and the more Ly $\alpha$ absorption it will produce. However, extracting quantitative mass loss measurements from the data requires the assistance of 
Table 1: Published Astrospheric Detections and Mass Loss Measurements

\begin{tabular}{|lcccccccc|}
\hline Star & $\begin{array}{c}\text { Spectral } \\
\text { Type }\end{array}$ & $\begin{array}{c}d \\
(\mathrm{pc})\end{array}$ & $\begin{array}{c}\text { Surf. Area } \\
\left(A_{\odot}\right)\end{array}$ & Log $\mathrm{L}_{x}$ & $\begin{array}{c}V_{\text {ISM }} \\
\left(\mathrm{km} \mathrm{s}^{-1}\right)\end{array}$ & $\begin{array}{c}\theta \\
(\mathrm{deg})\end{array}$ & $\begin{array}{c}\dot{M} \\
\left(\dot{M}_{\odot}\right)\end{array}$ & Reference \\
\hline$\alpha$ Cen & G2 V+K0 V & 1.3 & 2.22 & 27.70 & 25 & 79 & 2 & $1,2,3$ \\
Prox Cen & M5.5 V & 1.3 & 0.023 & 27.22 & 25 & 79 & $<0.2$ & 3 \\
$\epsilon$ Eri & K1 V & 3.2 & 0.61 & 28.32 & 27 & 76 & 30 & 4,10 \\
61 Cyg A & K5 V & 3.5 & 0.46 & 27.45 & 86 & 46 & 0.5 & 5,10 \\
$\epsilon$ Ind & K5 V & 3.6 & 0.56 & 27.39 & 68 & 64 & 0.5 & $6,7,8$ \\
EV Lac & M3.5 V & 5.1 & 0.123 & 28.99 & 45 & 84 & 1 & 11,12 \\
70 Oph & K0 V+K5 V & 5.1 & 1.32 & 28.49 & 37 & 120 & 100 & 11,12 \\
36 Oph & K1 V+K1 V & 6.0 & 0.88 & 28.34 & 40 & 134 & 15 & 9,10 \\
$\xi$ Boo & G8 V+K4 V & 6.7 & 1.00 & 28.90 & 32 & 131 & 5 & 11,12 \\
61 Vir & G5 V & 8.5 & 1.00 & 26.87 & 51 & 98 & 0.3 & 11,12 \\
$\delta$ Eri $^{a}$ & K0 IV & 9.0 & 6.66 & 27.05 & 37 & 41 & 4 & 11,12 \\
HD 128987 & G6 V & 24 & 0.71 & 28.60 & 8 & 79 & $?$ & 11,12 \\
$\lambda$ And $^{a}$ & G8 IV-III+M V & 26 & 55 & 30.53 & 53 & 89 & 5 & $6,7,8$ \\
DK UMa $^{a}$ & G4 III-IV & 32 & 19.4 & 30.36 & 43 & 32 & 0.15 & 11,12 \\
\hline
\end{tabular}

$a$ Uncertain detection.

References: (1) Linsky and Wood (1996). (2) Gayley et al. (1997). (3) Wood et al. (2001). (4) Dring et al. (1997). (5) Wood and Linsky (1998). (6) Wood et al. (1996). (7) Müller et al. (2001a). (8) Müller et al. (2001b). (9) Wood et al. (2000a). (10) Wood et al. (2002). (11) Wood et al. (2005b). (12) Wood et al. (2005a).

astrospheric models analogous to the heliospheric models discussed in Section 2.3.

The first step in modeling an astrosphere is to determine what the interstellar wind is like in the rest frame of the star. The proper motions and radial velocities of the nearby stars in Table 1 are known very accurately, as are their distances (see Perryman et al., 1997). The ISM flow vector is generally assumed to be the same as the LIC flow vector seen by the Sun (Lallement et al., 1995). Although multiple ISM velocity components are often seen towards even very nearby stars, the components are never separated by more than $5-10 \mathrm{~km} \mathrm{~s}^{-1}$, meaning that the LIC vector should be a reasonable approximation for these other clouds. An example is the nearby "G" cloud. Since $\alpha$ Cen, 70 Oph, and 36 Oph are known to lie within this cloud rather than the LIC, the "G" cloud vector from Lallement and Bertin (1992) is used instead of the LIC vector, but this does not change things very much. In any case, Table 1 lists the ISM wind velocity seen by each star $\left(V_{\text {ISM }}\right)$. The $\theta$ value in Table 1 indicates the angle between our line of sight to the star and the upwind direction of the ISM flow seen by the star.

Astrospheric models are extrapolated from a heliospheric model that fits the observed heliospheric Ly $\alpha$ absorption. The principle heliospheric model used in the past for this purpose is the four-fluid model described in Section 4.2, which is the source of the predicted heliospheric absorption in Figure 8. In order to convert this to an astrospheric model, the model is recomputed using the same wind and ISM input parameters, except for the ISM wind speed which is changed to the $V_{\text {ISM }}$ value appropriate for the star (see Table 1). The stellar wind proton density is varied to experiment with mass loss rates different from that of the Sun. Figure 9 shows four models of the $\alpha$ Cen astrosphere computed assuming different mass loss rates in the range of $\dot{M}=0.2-2 \dot{M}_{\odot}$ (Wood et al., 2001). The model astrospheres naturally become larger as the mass loss is increased. The figure shows the H I density distribution, but the models also provide temperature distributions and flow patterns. From these models astrospheric absorption can be computed for the $\theta=79^{\circ}$ line of sight to the star, for comparison with the data. Figure 10 shows this comparison

Living Reviews in Solar Physics

http://www. livingreviews . org/lrsp-2004-2 


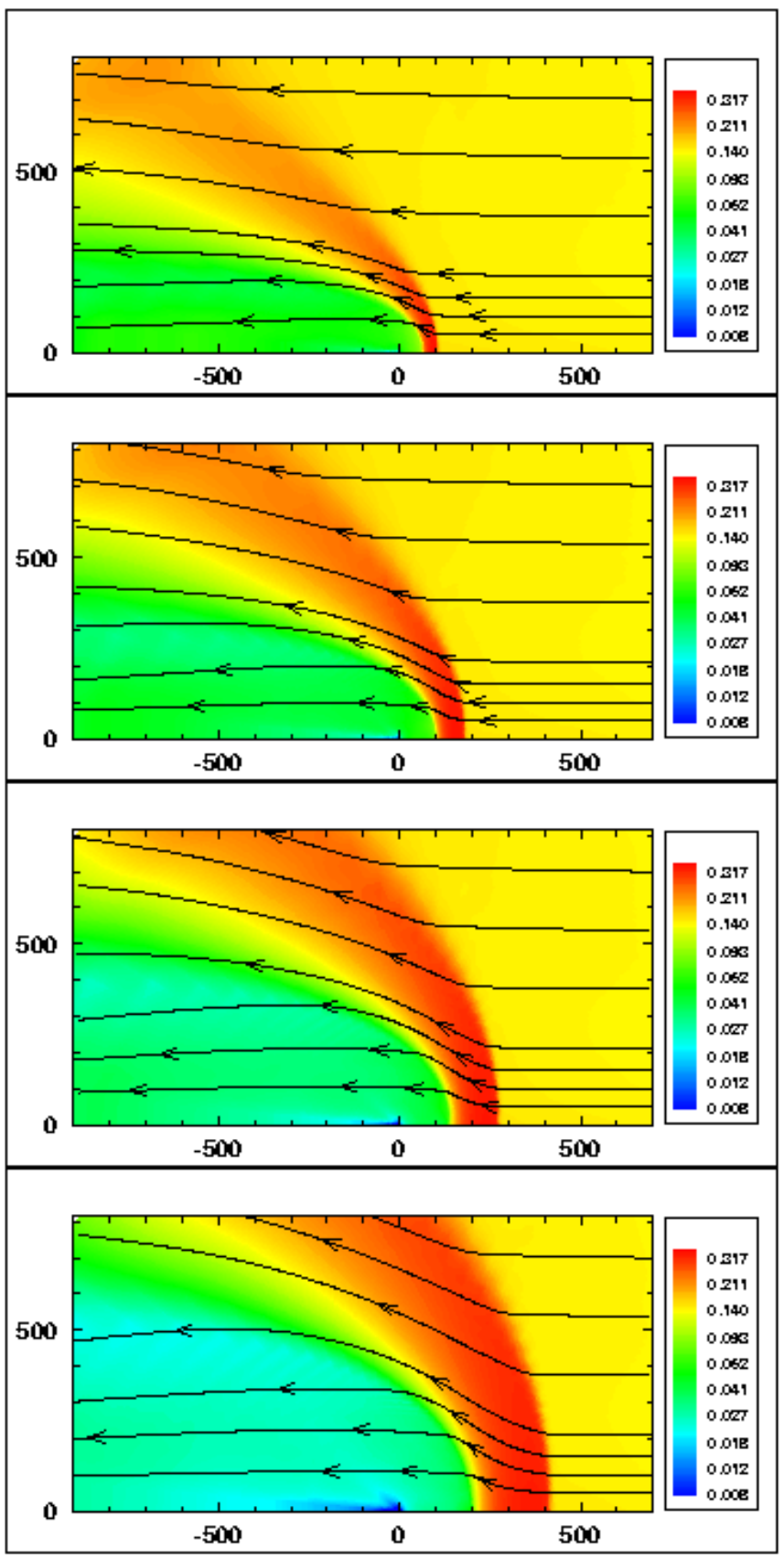

Figure 9: Distribution of $H$ I density predicted by hydrodynamic models of the Alpha/Proxima Cen astrospheres, assuming stellar mass loss rates of (from top to bottom) $0.2 \dot{M}_{\odot}, 0.5 \dot{M}_{\odot}, 1.0 \dot{M}_{\odot}$, and $2.0 \dot{M}_{\odot}$ (Wood et al., 2001). The distance scale is in AU. Streamlines show the H I flow pattern. 
for $\alpha$ Cen, along with similar comparisons for five other stars in Table 1 (Wood et al., 2002). The model with $\dot{M}=2 \dot{M}_{\odot}$ agrees best with the data, so this is the estimated mass loss rate of $\alpha$ Cen. Figure 11 shows similar data-model comparisons for six additional stars from Table 1 (Wood et al., 2005a).

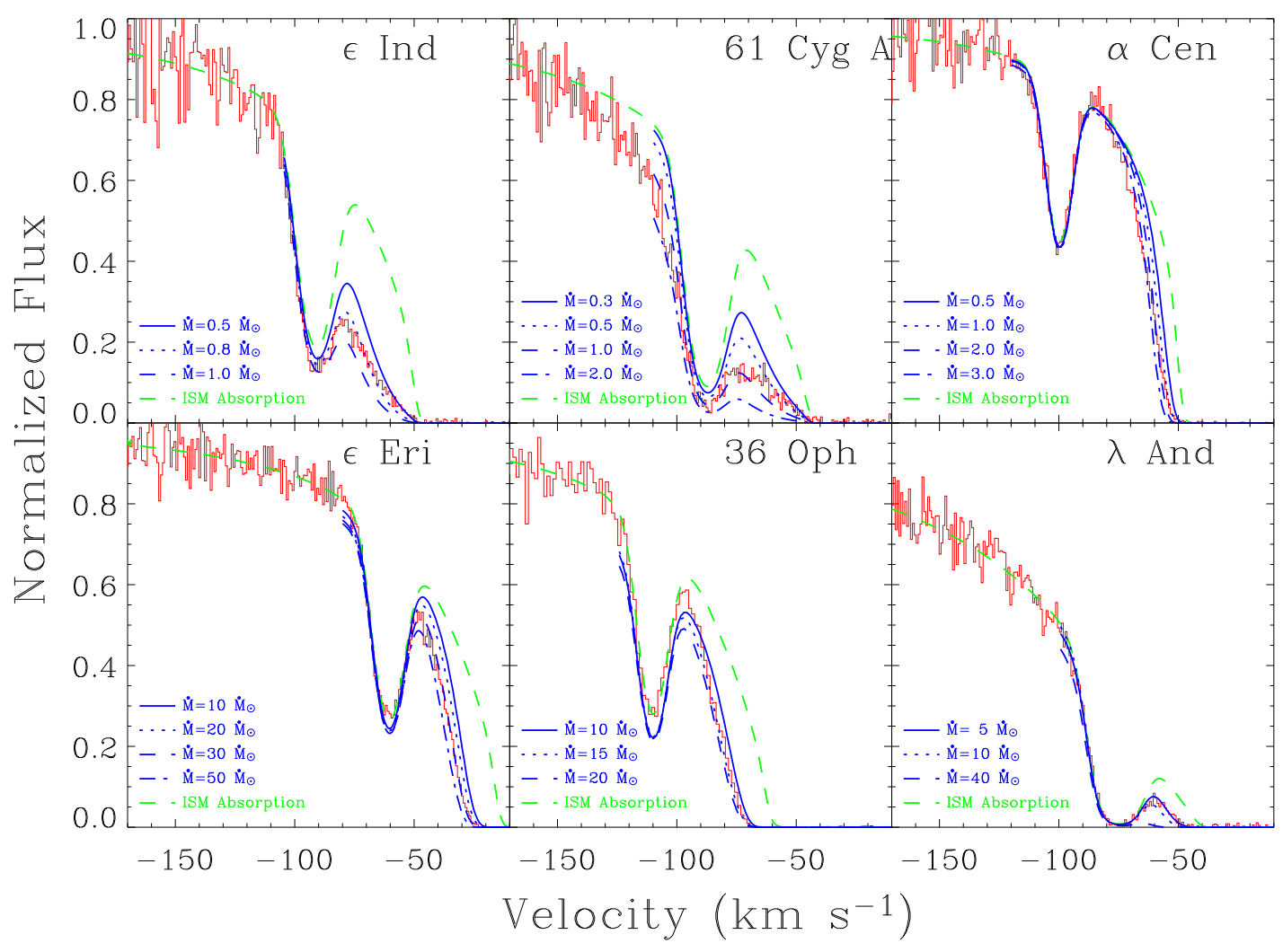

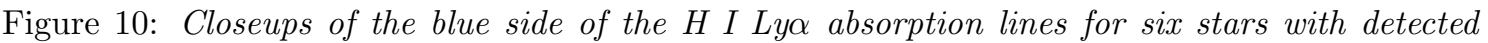
astrospheric absorption, plotted on a heliocentric velocity scale. Narrow D I ISM absorption is visible in all the spectra just blueward of the saturated $H$ I absorption. Green dashed lines indicate the interstellar absorption alone, and blue lines in each panel show the additional astrospheric absorption predicted by hydrodynamic models of the astrospheres assuming various mass loss rates (Wood et al., 2002).

Table 1 lists all mass loss rates measured in this manner, and Figures 12 and 13 show the astrospheric models that lead to the best fits to the data. The astrospheres vary greatly in size, both due to different mass loss rates and to different ISM wind speeds. It is worth noting that the largest of these astrospheres ( $\epsilon$ Eri, $70 \mathrm{Oph}$ ) would be comparable in size to the full Moon in the night sky, if we could see them.

Given the model dependence and other difficulties described in Section 4.2, one might wonder if mass loss rates derived using the astrospheric models are at all reliable. However, the crucial point is that the heliospheric model from which the astrospheric models are extrapolated successfully reproduces the heliospheric absorption. For the astrospheric modeling purposes described here, this is more important than whether the input parameters of that model are actually correct.

Living Reviews in Solar Physics

http: //www . livingreviews . org/lrsp-2004-2 


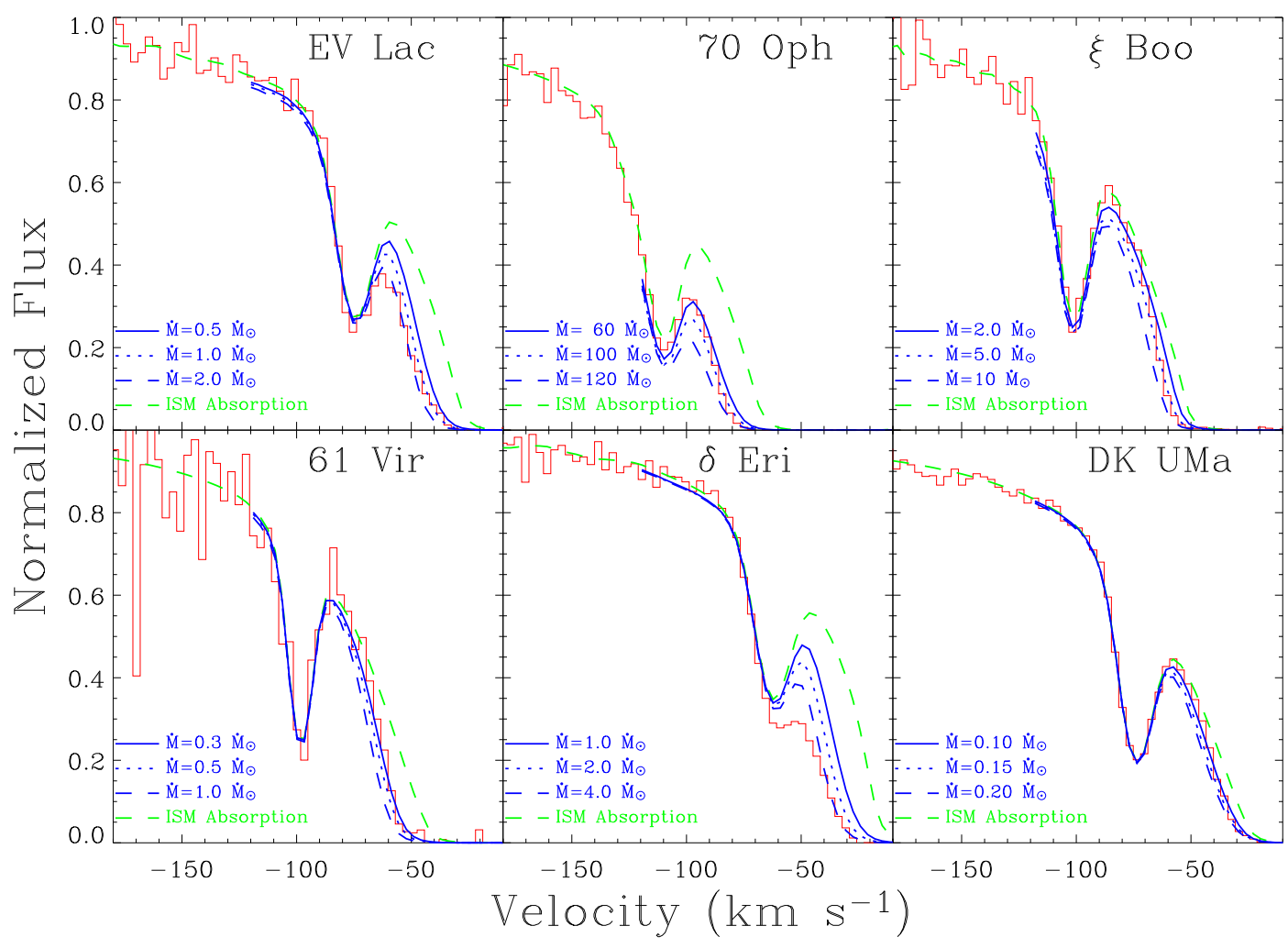

Figure 11: A figure analogous to Figure 10, but for six other lines of sight (Wood et al., 2005a). 


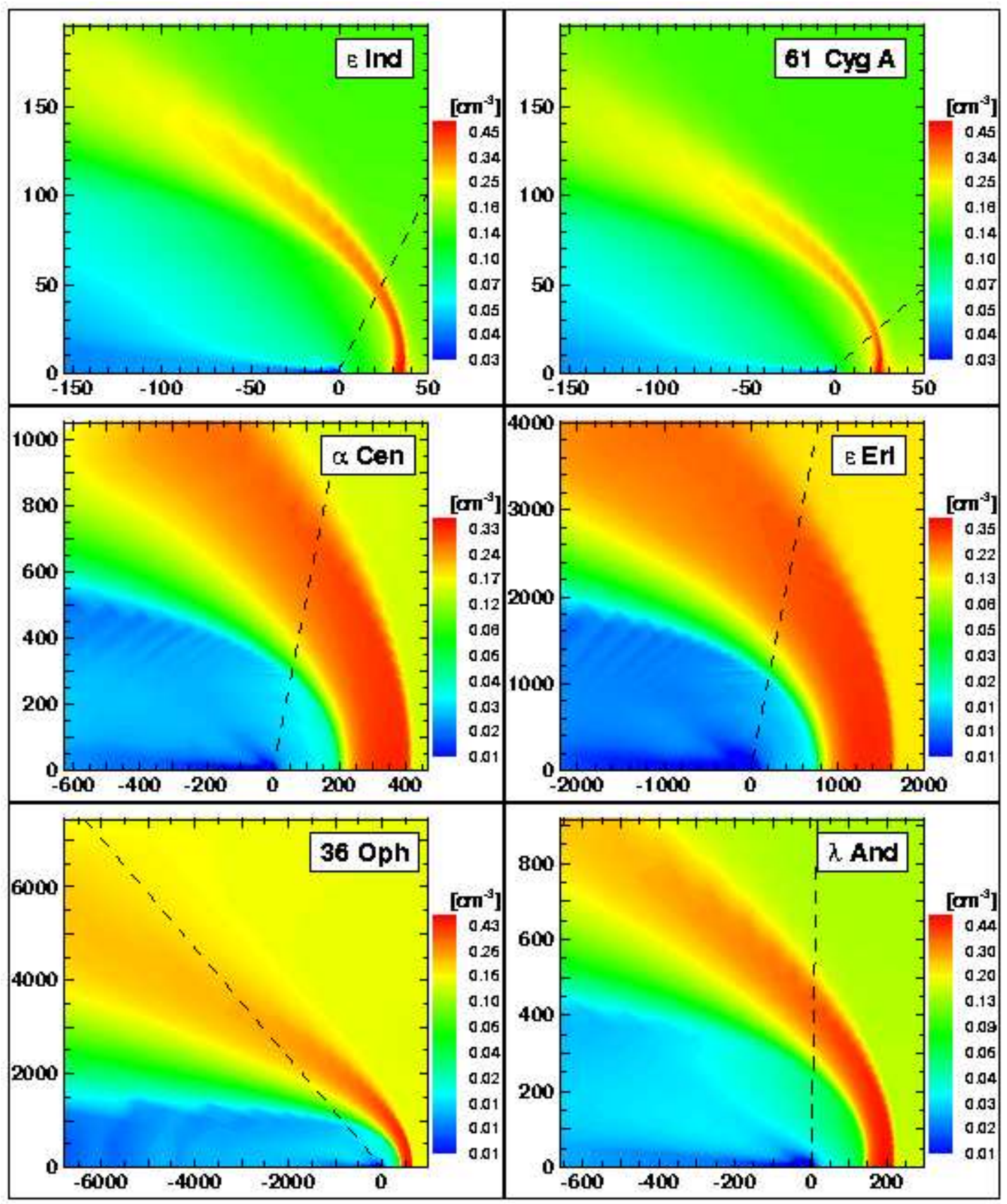

Figure 12: Maps of H I density from hydrodynamic models of stellar astrospheres (Wood et al., 2002). The models shown are the ones that lead to the best fits to the data in Figure 10. The distance scale is in $A U$. The star is at coordinate (0,0) and the ISM wind is from the right. The dashed lines indicate the Sun-star line of sight. 


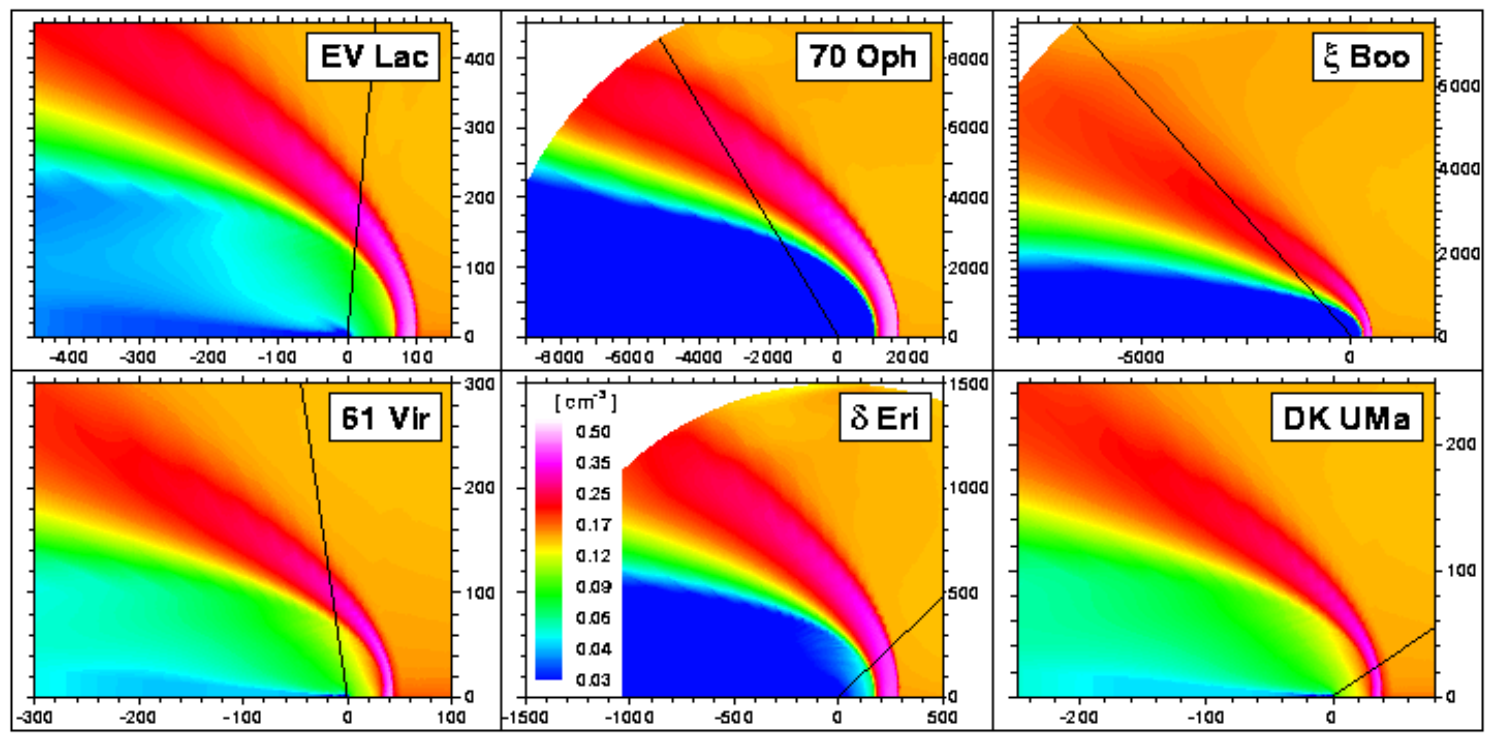

Figure 13: Maps of $H$ I density from hydrodynamic models of stellar astrospheres (Wood et al., 2005a), analogous to Figure 12. The models shown are the ones that lead to the best fits to the data in Figure 11.

In essence, the observed heliospheric absorption is calibrating the models before they are being applied to modeling astrospheres, and in this way the mass loss measurement technique is actually semi-empirical. Nevertheless, the mass loss rates measured using the astrospheric Ly $\alpha$ absorption technique should not be considered to be terribly precise. Uncertainties in the mass loss rates are probably of order a factor of 2 (Wood et al., 2002).

Other assumptions are implicitly made in this mass loss measurement procedure. One is that the LISM does not vary greatly from one star to the next. However, for these very nearby stars LISM variations should be modest, and as discussed in Section 4.2 modest variations probably do not greatly affect the predicted astrospheric absorption. Another assumption is that the stellar wind speeds of the stars in Table 1 are similar to that of the Sun. An argument in favor of this assumption is that stellar wind velocities are generally not very different from the surface escape speeds based on past experience, regardless of the type of wind or star one is considering (see Section 2.1). This includes the Sun, which has a surface escape speed of $619 \mathrm{~km} \mathrm{~s}^{-1}$, very similar to observed solar wind velocities. All the main sequence stars in Table 1 have similar escape speeds, so one might expect them to have similar wind velocities. However, the magneto-centrifugal wind acceleration models of Holzwarth and Jardine (2007) suggest that rapidly rotating stars might have wind speeds much faster than their surface escape speeds. Thus, the appropriateness of the constant wind speed assumption is still a matter for debate. The one star in Table 1 that has an escape velocity significantly different from that of the Sun is $\lambda$ And. The G8 IV-III primary that surely dominates the wind from this binary is not a main sequence star, and it has a surface escape speed about 3 times lower than that of the Sun. Perhaps in the future the mass loss rate listed in Table 1 should be remeasured assuming a lower wind velocity.

In addition to the mass loss measurements for stars with detected astrospheric absorption, Table 1 also lists an upper limit derived from a nondetection for Proxima Cen. Upper limits cannot be computed for most stars with nondetections, because a likely interpretation for most nondetections is that the star is surrounded by hot, fully ionized ISM material, rather than partially 
neutral gas like that which surrounds the Sun. The Local Bubble in which the Sun is located is mostly filled with this hot material (see Section 2.2). The Sun just happens to be located within one of the cooler, partially neutral clouds that lie within the Bubble. For Proxima Cen, the hot ISM explanation for the astrospheric nondetection can be discarded, because Proxima Cen's distant companion $\alpha$ Cen has detected astrospheric absorption, proving that Proxima Cen is not located within the hot ISM. Thus, for Proxima Cen a meaningful upper limit to the stellar mass loss rate can be derived.

No mass loss measurement is reported for HD 128987 in Table 1, since no astrospheric model seems to be able to reproduce the apparent astrospheric absorption, bringing into question the astrospheric interpretation of the absorption for this star (Wood et al., 2005a). The fundamental problem is the very low LISM wind speed seen by this $\operatorname{star}\left(V_{\mathrm{ISM}}=8 \mathrm{~km} \mathrm{~s}^{-1}\right.$; see Table 1$)$. This low speed is not sufficient to result in much deceleration and heating of neutral $\mathrm{H}$ within the astrosphere, thereby yielding little predicted absorption. Thus, the apparent astrospheric detection for HD 128987 remains a mystery at this time. 


\section{Implications for the Sun and Solar System}

\subsection{Inferring the mass loss history of the Sun}

In addition to stellar mass loss rates, Table 1 lists coronal X-ray luminosities from the ROSAT PSPC instrument (see Hünsch et al., 1999; Schmitt and Liefke, 2004). Solar-like winds have their origins in stellar coronae (see Section 2.1), so one might expect the winds to be correlated with coronal properties such as X-ray emission. Thus, in Figure 14 the mass loss rates measured from

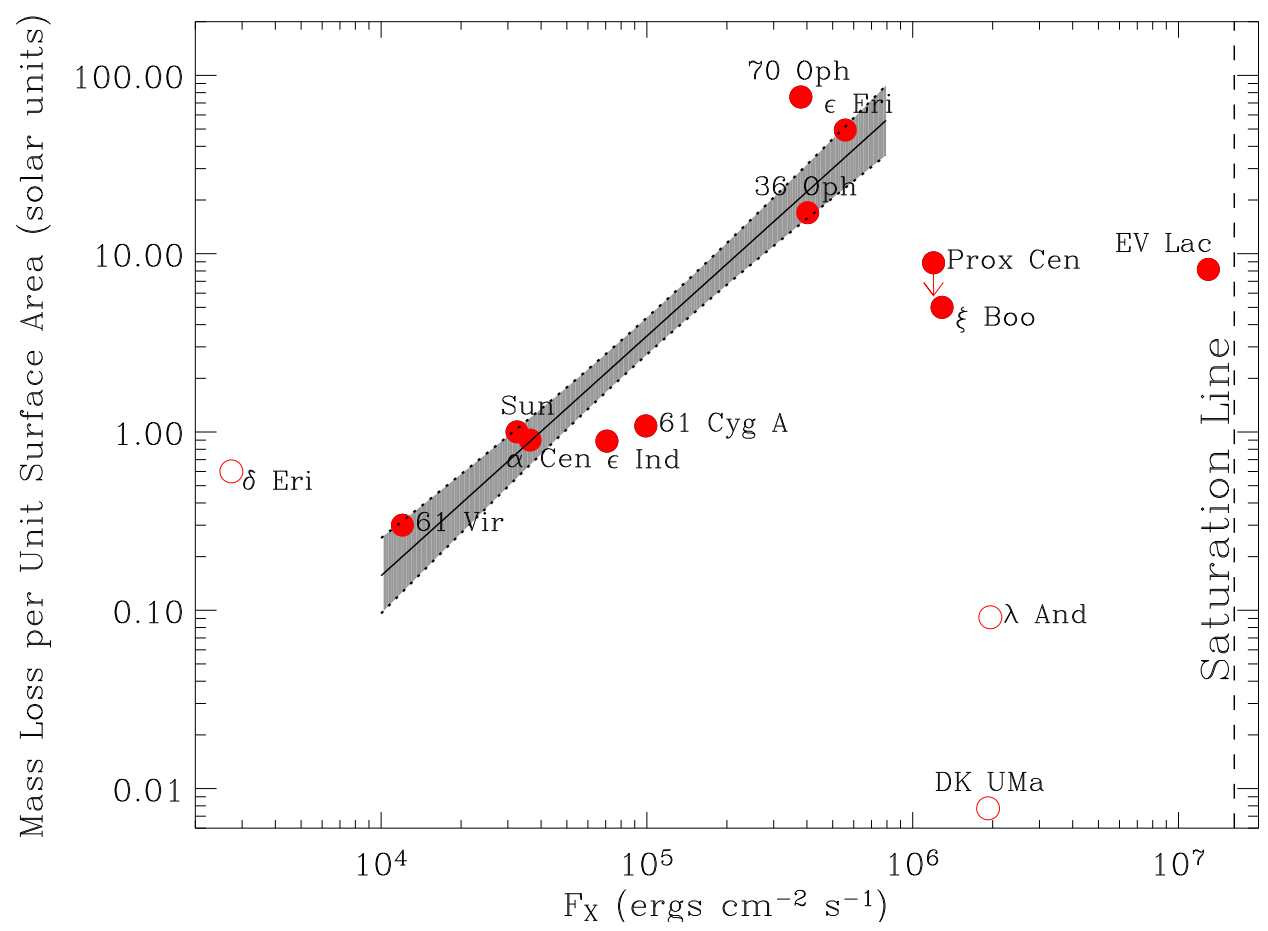

Figure 14: Measured mass loss rates (per unit surface area) plotted versus X-ray surface flux (Wood et al., 2005a). The filled and open circles are main sequence and evolved stars, respectively. For the main sequence stars with $\log F_{X}<8 \times 10^{5} \mathrm{ergs} \mathrm{cm}^{-2} \mathrm{~s}^{-1}$, mass loss appears to increase with coronal activity, so a power law has been fitted to these stars, and the shaded region is the estimated uncertainty in the fit. The saturation line represents the maximum $F_{\mathrm{X}}$ value observed from solar-like stars.

the astrospheric Ly $\alpha$ absorption (per unit surface area) are plotted versus X-ray surface fluxes (Wood et al., 2005a). For the main sequence stars mass loss increases with coronal activity. A power law is fitted to these GK stars in Figure 14. Quantitatively, this relation is

$$
\dot{M} \propto F_{\mathrm{X}}^{1.34 \pm 0.18} \text {. }
$$

The saturation line in Figure 14 indicates the maximum X-ray flux observed from solar-like stars (Güdel et al., 1997).

It is interesting to note that during the solar cycle, the Sun's wind strength is actually anticorrelated with its X-ray flux. The solar wind is weaker at solar maximum than at solar minimum despite coronal X-ray fluxes being much higher (Lazarus and McNutt Jr, 1990). This is presumably due to the fact that winds are more associated with the large scale dipole component of the 
solar magnetic field instead of the small scale active regions responsible for most of the Sun's X-ray emission. The dipole field actually weakens at solar maximum along with the wind. However, the interior magnetic dynamo is ultimately responsible for both the small scale and large scale fields, so as a whole both field components should increase with increasing dynamo activity, consistent with the mass loss/activity correlation in Figure 14 (Schrijver et al., 2003).

The evolved stars are clearly inconsistent with the main sequence stars in Figure 14 . The very active coronae of $\lambda$ And and DK UMa produce surprisingly weak winds, though it should be noted that both of these astrospheric detections are flagged as being questionable in Table 1. There are three main sequence stars with $\log F_{X}>8 \times 10^{5} \mathrm{ergs} \mathrm{cm}^{-2} \mathrm{~s}^{-1}$, which have low mass loss measurements that are not consistent with the wind-activity correlation that seems to exist for the low activity main sequence stars. Two of these stars (Proxima Cen and EV Lac) are tiny $\mathrm{M}$ dwarf stars. If these were the only discrepant data points one could perhaps argue that the discrepancy is due to these $\mathrm{M}$ dwarfs being significantly less solar-like than the $\mathrm{G}$ and $\mathrm{K}$ dwarfs that make up the rest of the main sequence sample of stars. However, this interpretation is invalidated by the third discrepant measurement, that of $\xi$ Boo. Being a binary with two rather solar-like stars $(\mathrm{G} 8 \mathrm{~V}+\mathrm{K} 4 \mathrm{~V})$, there is no easy way to dismiss the $\xi$ Boo measurement, which implies that the power law relation does not extend to high activity levels for any type of star. More mass loss measurements of active stars would clearly be helpful to better define the characteristics of solar-like winds at high coronal activity levels.

Based on the available data, the mass-loss/activity relation appears to change its character at $\log F_{X} \approx 8 \times 10^{5} \mathrm{ergs} \mathrm{cm}^{-2} \mathrm{~s}^{-1}$. One possible explanation for this concerns the existence of polar spots for very active stars. Low activity stars presumably have starspot patterns like that of the Sun, where spots are confined to low latitudes. However, for very active stars not only are spots detected at high latitudes, but a majority of these stars show evidence for large polar spots (Strassmeier, 2002). The existence of high latitude and polar spots represents a fundamental change in the stellar magnetic geometry (Schrijver and Title, 2001), and it is possible that this dramatic change in magnetic field structure could affect the winds emanating from these stars. Perhaps stars with polar spots might have a magnetic field with a strong dipolar component that could envelope the entire star and inhibit stellar outflows, thereby explaining why active stars have weaker winds than the mass-loss/activity relation of less active main sequence stars would predict. For $\xi$ Boo A, high latitude spots of some sort have been detected (Toner and Gray, 1988). Petit et al. (2005) have detected a strong global dipole field component for $\xi$ Boo A, consistent with the picture presented above. They also detected a large-scale toroidal field component, which would have no solar analog whatsoever, consistent with the idea that very active solar-like stars have significantly different magnetic field structures from those of the Sun and other low-activity stars.

Figure 14 illustrates how mass loss varies with coronal activity. But what about age? There is a known connection between activity and age, for the following reasons. The gravitational contraction of interstellar clouds that results in star formation leads to rapid rotation for young, newly born stars. This rapid rotation leads to vigorous dynamo activity and therefore high surface magnetic activity and high coronal X-ray emission. However, the magnetic fields of these young, rapidly rotating stars drag against their winds, and this magnetic braking gradually slows the stellar rotation. This in turn leads to lower activity levels and X-ray fluxes. An enormous amount of effort has been expended in the past few decades to observationally establish exactly how rotation relates to stellar age (see Skumanich, 1972; Soderblom et al., 1993) and how rotation relates to stellar activity, which is most easily measured through X-ray emission (see Pallavicini et al., 1981; Walter, 1982, 1983; Caillault and Helfand, 1985; Micela et al., 1985; Fleming et al., 1989; Stauffer et al., 1994). For solar-like stars, Ayres (1997) finds

$$
V_{\text {rot }} \propto t^{-0.6 \pm 0.1}
$$

Living Reviews in Solar Physics

http://www. livingreviews . org/lrsp-2004-2 
for the rotation/age relation, while X-ray flux and rotation are related by

$$
F_{\mathrm{X}} \propto V_{\text {rot }}^{2.9 \pm 0.3}
$$

Equations (1), (2), and (3) can be combined to obtain the following relation between mass loss and age for solar-like stars:

$$
\dot{M} \propto t^{-2.33 \pm 0.55} \text {. }
$$

This is the first empirically determined mass loss evolution law for solar-like stars, and Figure 15 shows what this relation implies for the mass loss history of the Sun in particular (Wood et al., 2005a).

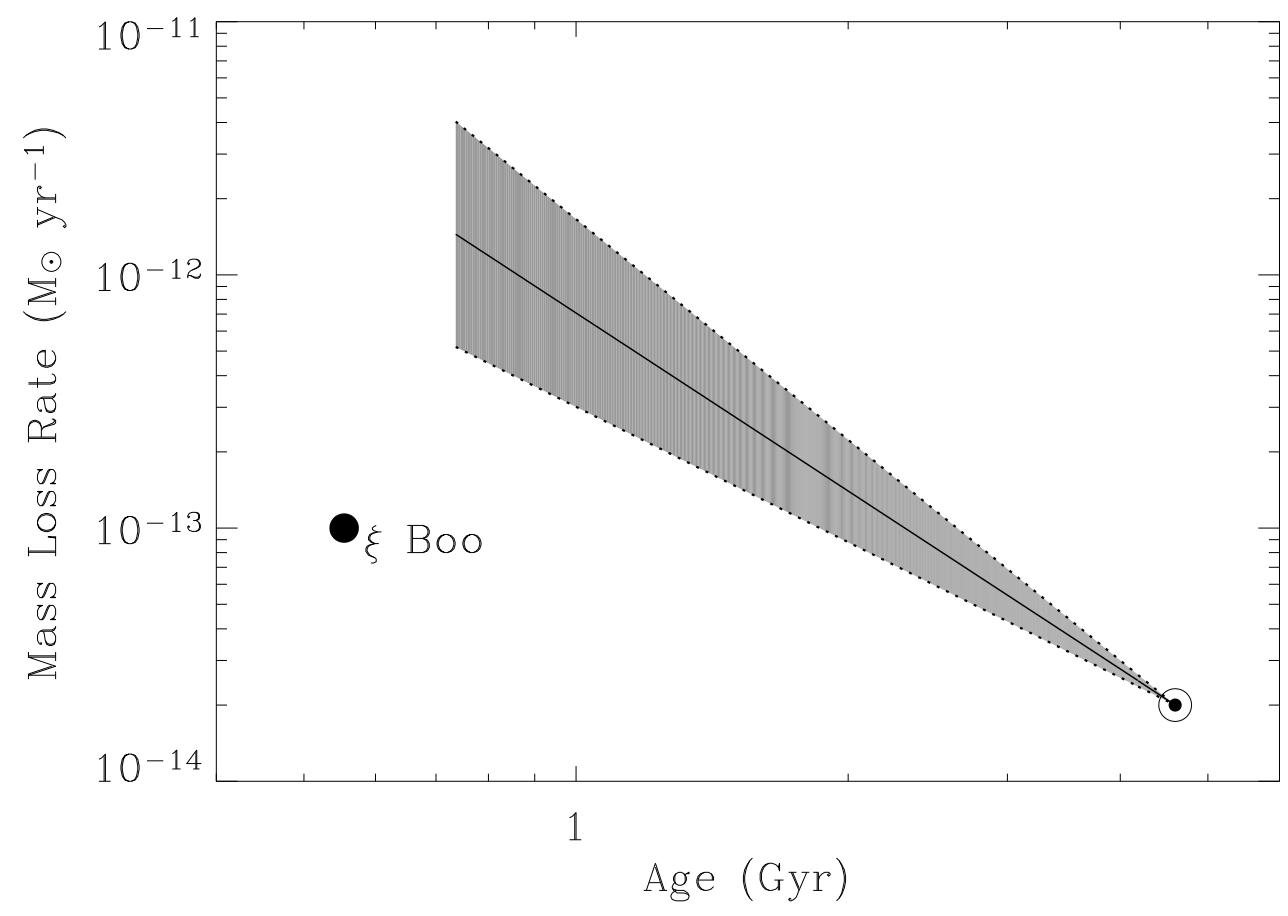

Figure 15: The mass loss history of the Sun suggested by the power law relation from Figure 14 (Wood et al., 2005a). The low mass-loss rate measurement for $\xi$ Boo implies that the wind weakens at $t \approx 0.7 \mathrm{Gyr}$ as one goes back in time.

The truncation of the power law relation in Figure 14 leads to the truncation of the massloss/age relation in Figure 15 at about $t=0.7 \mathrm{Gyr}$. The location of $\xi$ Boo is shown in order to infer what the solar wind might have been like at earlier times. Despite the high activity cutoff, the mass loss measurements obtained so far clearly suggest that winds are generally stronger for young solar-like stars, and as a consequence the solar wind was presumably much stronger early in the Sun's lifetime. This has many important implications, some of which are discussed in Sections 5.2, 5.3 , and 5.4 .

\subsection{Magnetic braking}

The magnetic braking process by which stars like the Sun shed angular momentum is the reason why stellar activity decreases with time and why the solar mass loss/age relation in Figure 15 
can be inferred from the mass loss/activity relation in Figure 14 (see Section 5.1). Winds play an important role in this process, because it is the wind that the stellar field drags against in slowing down the star's rotation. The efficiency of this braking is clearly related to the density of the wind, and therefore to the mass loss rate. Thus, the wind evolution law in Equation (4) has consequences for how the effectiveness of the magnetic braking changes with time. Models for magnetic braking suggest relations of the form

$$
\frac{\dot{\Omega}}{\Omega} \propto \frac{\dot{M}}{M}\left(\frac{R_{\mathrm{A}}}{R}\right)^{m},
$$

where $\Omega$ is the angular rotation rate and $R_{\mathrm{A}}$ is the Alfvén radius (Weber and Davis Jr, 1967; Stepień, 1988; Gaidos et al., 2000). The exponent $m$ is a number between 0 and 2 , where $m=2$ corresponds to a purely radial magnetic field. Mestel (1984) claims that more reasonable magnetic geometries suggest $m=0-1$. The Alfvén radius is

$$
R_{\mathrm{A}}=\sqrt{\frac{V_{\mathrm{w}} \dot{M}}{B_{\mathrm{r}}^{2}}},
$$

where $V_{\mathrm{w}}$ is the stellar wind speed and $B_{\mathrm{r}}$ is the disk-averaged radial magnetic field.

For a star like the Sun, the star's mass and radius are relatively invariant. If $V_{\mathrm{w}}$ does not vary with time, which was also an assumption used in the derivation of mass loss rates from the astrospheric absorption (see Section 4.3), then the time dependence of all quantities in Equations (5) and (6) are known except for that of $B_{\mathrm{r}}$. If $B_{\mathrm{r}}$ is expressed as a power law, $B_{\mathrm{r}} \propto t^{\alpha}$, Equations (4), (5), and (6) combined suggest

$$
\alpha=1 / m-(1.17 \pm 0.28)(m+2) / m .
$$

Assuming that $m$ is in the physically allowable range of $m=0-2$ yields the upper limit $\alpha<-1.3$, while the more likely range of $m=0-1$ suggested by Mestel (1984) implies $\alpha<-1.7$. In any case, the empirical mass loss evolution law in Equation (4) is consistent with theoretical descriptions of magnetic braking only if disk-averaged stellar magnetic fields decline at least as fast as $t^{-1.3}$.

\subsection{The Faint Young Sun problem}

Evolutionary models of the solar interior lead to the prediction that the Sun should have been about 25\% fainter 3.8 Gyr ago (Gough, 1981; Bahcall et al., 2001). This is a fairly robust result that has been known for a long time. However, this creates significant difficulties for those who study the climate history of planets in our solar system. With the young Sun this faint, the planets should have been significantly colder than they are now. On Earth and Mars, surface water should have been frozen, but this contradicts geologic evidence that abundant water existed and flowed on the surfaces of both of these planets. This is the so-called "Faint Young Sun" paradox (Sagan and Mullen, 1972; Kasting, 1991). Most attempts to solve this problem have appealed to increased amounts of greenhouse gases in the early atmospheres of Earth and Mars, which allowed the surface temperatures to remain similar to present-day temperatures despite a fainter Sun (see Walker, 1985; Kasting and Ackerman, 1986; Kasting, 1991).

However, a couple theories have been advanced that involve the solar wind. One of these is simply that the young solar wind was strong enough to significantly decrease the mass of the Sun, meaning that the young Sun was more massive and thus more luminous than standard solar evolutionary models predict (Guzik et al., 1987; Sackmann and Boothroyd, 2003). If the Sun was only about $2 \%$ more massive $3.8 \mathrm{Gyr}$ ago, then the Sun would still have been bright enough to maintain sufficiently warm temperatures on Earth and Mars. The mass loss evolution law derived from stellar wind measurements in Equation (4) does indeed suggest that the solar wind was

Living Reviews in Solar Physics

http://www. livingreviews.org/lrsp-2004-2 
stronger in the past. Unfortunately, it was not strong enough. If Equation (4) is correct, the Sun could not have been more than $0.2 \%$ more massive 3.8 Gyr ago. Thus, this theory ultimately is not supported by the stellar wind measurements (Minton and Malhotra, 2007).

However, there is a less direct method that has been proposed by which a stronger young solar wind could contribute to a solution of the Faint Young Sun problem. There have been several claims that cosmic rays have important effects on the Earth's climate by stimulating cloud formation, which is believed to generally cool the Earth's atmosphere (see Svensmark, 1988). However, cosmic rays are modulated by the solar wind, and a stronger solar wind would reduce the flux of cosmic rays into the Earth's atmosphere. Thus, the idea is that the stronger wind of the young Sun led to lower cosmic ray fluxes, helping the Earth (and perhaps Mars) to maintain warm temperatures (Shaviv, 2003). However, the whole idea of cosmic rays influencing the terrestrial climate to this degree remains very controversial (see Carslaw et al., 2002).

\subsection{Erosion of planetary atmospheres}

Even if the solar wind plays no role in the solution to the Faint Young Sun paradox described in Section 5.3, the solar wind may still have had a dramatic effect on planets in the past through the eroding effects of the wind on planetary atmospheres. Solar wind sputtering processes have been proposed as having had important effects on the atmospheres of both Venus and Titan (Chassefière, 1997; Lammer et al., 2000), but it is the Martian atmosphere that may have been the most dramatically affected by the solar wind. The connection between the Martian atmosphere and the question of whether life once existed on the planet makes the Mars example even more interesting.

Mars apparently had running water on its surface in the distant past, but it is now very dry. Isotopic evidence strongly suggests that the Martian atmosphere was once much thicker and more conducive to the existence of surface water (see Carr, 1996; Jakosky and Phillips, 2001). What caused the loss of the early Martian atmosphere, and presumably the loss of surface water and habitability as well? Solar wind sputtering is a leading candidate for the cause of these changes (Luhmann et al., 1992; Perez de Tejada, 1992; Jakosky et al., 1994; Kass and Yung, 1995; Lundin, 2001). Unlike Earth, the Martian atmosphere is not currently protected from the solar wind by a strong magnetosphere. There is evidence that Mars once had a magnetic field, but it disappeared at least $3.9 \mathrm{Gyr}$ ago (Acuña et al., 1999). At that point, Mars would have been exposed to a solar wind about 80 times stronger than the current wind according to Figure 15. The stronger solar wind makes wind erosion an even more likely culprit behind the disappearance of most of the Martian atmosphere.

Studies of Martian atmosphere evolution that consider the wind evolution law inferred from the astrospheric measurements, along with modern estimates of the history of solar X-ray and UV fluxes, have already begun (Guinan et al., 2003; Lammer et al., 2003; Ribas et al., 2005). Investigations into how the solar wind may have affected other planets will undoubtedly also be an active area of future research, stimulated in part by the discovery of planets around other stars. Many of these extrasolar planets orbit very close to their stars, which means that they will be exposed to wind fluxes orders of magnitude higher than the Earth or Mars have ever seen. Knowledge of the evolution of solar-like winds is crucial for understanding the atmospheric evolution of these extrasolar planets, and efforts are already underway to try to model wind erosion effects on such planets (Grießmeier et al., 2004; Preusse et al., 2005). 


\section{Conclusions}

The study of solar-like winds around other stars is not an easy one, due to the difficulty in detecting these winds. However, such measurements are the only way to empirically infer the history of the solar wind and to assess its potential effects on atmospheres of planets in our solar system. At this time, the indirect astrospheric Ly $\alpha$ absorption technique is the only way to detect weak solar-like stellar winds. Radio and X-ray observations can place limits on wind fluxes (see Section 3), but there is no reason to believe that these diagnostics will approach the sensitivity level of the Ly $\alpha$ diagnostic in the near future.

Currently there are only about a dozen mass loss measurements for solar-like stars, a rather small data sample. Additional astrospheric detections are required to better constrain relations between stellar winds, activity, and age. Unfortunately, the STIS instrument on HST failed in August 2004, and at the time of this writing is still unavailable. Furthermore, there is no future mission even in the planning stages that would be capable of the high resolution UV spectrometry necessary to detect absorption signatures of stellar astrospheres. Thus, the future of this subject area is very uncertain, at least on the data side. On the theory side, there is hope that improvements in our ability to numerically model the heliosphere and astrospheres will allow more precise analyses of existing data, and in this way improve our understanding of solar-like stellar winds and astrospheres. 


\section{Acknowledgements}

I would like to thank my primary collaborators Jeff Linsky, Hans Müller, Gary Zank, and Vlad Izmodenov for their contributions to the astrospheric Ly $\alpha$ research. I would also like to thank David McComas and Rosine Lallement for providing figures for this article, and Priscilla Frisch for fruitful discussions. Support for this work was provided by NASA through grants NAG5-9041 and NNG05GD69G to the University of Colorado, and through grant number AR-09957 from the Space Telescope Science Institute, which is operated by AURA, Inc., under NASA contract NAS5-26555. Extensive use of NASA's Astrophysics Data System Bibliographic Services has been made in the preparation of this article. 


\section{References}

Acuña, M.H., Connerney, J.E.P., Ness, N.F., Lin, R.P., Mitchell, D., Carlson, C.W., McFadden, J., Anderson, K.A., Rème, H., Mazelle, C., Vignes, D., Wasilewski, P., Cloutier, P., 1999, "Global Distribution of Crustal Magnetization Discovered by the Mars Global Surveyor MAG/ER Experiment", Science, 284, 790-793 5.4

Ayres, T.R., 1997, "Evolution of the Solar Ionizing Flux", J. Geophys. Res., 102, 1641-1652 5.1

Bahcall, J.N., Pinsonneault, M.H., Basu, S., 2001, "Solar Models: Current Epoch and Time Dependences, Neutrinos, and Helioseismological Properties", Astrophys. J., 555, 990-1012. Related online version (cited on 13 May 2004):

http: //adsabs.harvard.edu/abs/2001ApJ . .555. .990B 5.3

Baranov, V.B., 1990, "Gasdynamics of the Solar Wind Interaction with the Interstellar Medium", Space Sci. Rev., 52, 89-120 2.3

Baranov, V.B., Malama, Y.G., 1993, "Model of the solar wind interaction with the local interstellar medium - Numerical solution of self-consistent problem", J. Geophys. Res., 98, 15,157-15,163 $2.3,4.2$

Baranov, V.B., Malama, Y.G., 1995, "Effect of Local Interstellar Medium Hydrogen Fractional Ionization on the Distant Solar Wind and Interface Region", J. Geophys. Res., 100, 14,755$14,7622.3,4.2$

Baranov, V.B., Zaitsev, N.A., 1995, "On the Problem of the Solar Wind Interaction with Magnetized Interstellar Plasma", Astron. Astrophys., 304, 631-637. Related online version (cited on 13 May 2004):

http://adsabs.harvard.edu/abs/1995A\%26A...304..631B 2.3

Bertaux, J.-L., Blamont, J.E., 1971, "Evidence for a Source of an Extraterrestrial Hydrogen Lyman- $\alpha$ Emission: the Interstellar Wind", Astron. Astrophys., 11, 200-217. Related online version (cited on 13 May 2004):

http://adsabs.harvard.edu/abs/1971A\%26A...11..200B 2.2, 2.3

Biermann, L., 1951, "Kometenschweife und solare Korpuskularstrahlung", Z. Astrophys., 29, 274286. Related online version (cited on 13 May 2004):

http://adsabs.harvard.edu/abs/1951ZA....29. .274B 2.1

Boesgaard, A.M., Steigman, G., 1985, "Big Bang Nucleosynthesis: Theories and Observations", Annu. Rev. Astron. Astrophys., 23, 319-378. Related online version (cited on 13 May 2004): http://adsabs.harvard.edu/abs/1985ARA\%26A. .23. .319B 4.1

Bond, H.E., Mullan, D.J., O’Brien, M.S., Sion, E.M., 2001, "Detection of Coronal Mass Ejections in V471 Tauri with the Hubble Space Telescope", Astrophys. J., 560, 919-927. Related online version (cited on 13 May 2004):

http://adsabs.harvard.edu/abs/2001ApJ ...560..919B 3

Brown, A., Vealé, A., Judge, P., Bookbinder, J.A., Hubeny, I., 1990, "Stringent Limits on the Ionized Mass Loss from A and F Dwarfs", Astrophys. J., 361, 220-224. Related online version (cited on 13 May 2004):

http://adsabs.harvard.edu/abs/1990ApJ...361..220B 3

Living Reviews in Solar Physics

http: //www. livingreviews.org/lrsp-2004-2 
Burlaga, L.F., Ness, N.F., Stone, E.C., McDonald, F.B., Acuña, M.H., Lepping, R.P., Connerney, J.E.P., 2003, "Search for the Heliosheath with Voyager 1 Magnetic Field Measurements", Geophys. Res. Lett., 30, 2072-2075 2.3

Burles, S., Nollett, K.M., Turner, M.S., 2001, "Big Bang Nucleosynthesis Predictions for Precision Cosmology", Astrophys. J. Lett., 552, L1-L5. Related online version (cited on 13 May 2004): http://adsabs.harvard.edu/abs/2001ApJ...552L...1B 4.1

Caillault, J.-P., Helfand, D.J., 1985, "The Einstein Soft X-ray Survey of the Pleiades", Astrophys. J., 289, 279-299. Related online version (cited on 13 May 2004): http://adsabs.harvard.edu/abs/1985ApJ . . 289 . 279C 5.1

Carr, M.H., 1996, Water on Mars, Oxford University Press, New York, U.S.A. 5.4

Carslaw, K.S., Harrison, R.G., Kirkby, J., 2002, "Cosmic Rays, Clouds, and Climate", Science, 298, 1732-1737 5.3

Chassefière, E., 1997, "Loss of Water on the Young Venus: The Effect of a Strong Primitive Solar Wind", Icarus, 126, 229-232 5.4

Cheng, K.-P., Bruhweiler, F.C., 1990, "Ionization Processes in the Local Interstellar Medium: Effects of the Hot Coronal Substrate", Astrophys. J., 364, 573-581. Related online version (cited on 13 May 2004): http://adsabs.harvard.edu/abs/1990ApJ...364..573C 2.2

Chiappini, C., Renda, A., Matteucci, F., 2002, "Evolution of Deuterium, ${ }^{3} \mathrm{He}$ and ${ }^{4} \mathrm{He}$ in the Galaxy", Astron. Astrophys., 395, 789-801 4.1

Christensen-Dalsgaard, J., 2003, "Problems, Connections and Expectations of Asteroseismology", Astrophys. Space Sci., 284, 277-2941

Cranmer, S.R., 2002, "Coronal Holes and the High-Speed Solar Wind", Space Sci. Rev., 101, 229-294 2.1

Cravens, T.E., 2000, "Heliospheric X-ray Emission Associated with Charge Transfer of the Solar Wind with Interstellar Neutrals", Astrophys. J. Lett., 532, L153-L156. Related online version (cited on 13 May 2004):

http://adsabs.harvard.edu/abs/2000ApJ . . 532L.153C 3

Cravens, T.E., 2002, "X-ray Emission from Comets", Science, 296, 1042-1046 3

Dessler, A.J., 1967, "Solar Wind and Interplanetary Magnetic Field”, Rev. Geophys., 5, 11

Diplas, A., Savage, B.D., 1994, "An IUE survey of Interstellar H I Ly $\alpha$ Absorption. 1. Column Densities", Astrophys. J. Suppl. Ser., 93, 211-228. Related online version (cited on 13 May 2004): http://adsabs.harvard.edu/abs/1994ApJS. .93. .211D 4.1

Drake, S.A., Simon, T., Brown, A., 1993, "Detection of Radio Continuum Emission from Procyon", Astrophys. J., 406, 247-251. Related online version (cited on 13 May 2004): http://adsabs.harvard.edu/abs/1993ApJ...406. .247D 3

Dring, A.R., Linsky, J.L., Murthy, J., Henry, R.C., Moos, H.W., Vidal-Madjar, A., Audouze, J., Landsman, W.B., 1997, "Lyman-Alpha Absorption and the D/H Ratio in the Local Interstellar Medium", Astrophys. J., 488, 760-775. Related online version (cited on 13 May 2004): http://adsabs.harvard.edu/abs/1997ApJ . .488..760D 4.3 
Dupree, A.K., Baliunas, S.L., Shipman, H.L., 1977, "Deuterium and Hydrogen in the Local Interstellar Medium", Astrophys. J., 218, 361-369. Related online version (cited on 13 May 2004): http://adsabs.harvard.edu/abs/1977ApJ . . 218. .361D 4.1

Fahr, H.J., 1978, "Change of Interstellar Gas Parameters in Stellar-wind-dominated Astrospheres: Solar Case", Astron. Astrophys., 66, 103-117. Related online version (cited on 13 May 2004): http://adsabs.harvard.edu/abs/1978A\%26A...66. .103F 1

Favata, F., Micela, G., 2003, "Stellar Coronal Astronomy", Space Sci. Rev., 108, 577-708 1

Feldman, W.C., Asbridge, J.R., Bame, S.J., Gosling, J.T., 1977, "Plasma and Magnetic Fields from the Sun", in The Solar Output and its Variation, (Ed.) White, O.R., Proceedings of a Workshop, held in Boulder, Colorado, April 26-28, 1976, p. 351, Colorado Associated University Press, Boulder, U.S.A. 2.1

Fleming, T.A., Gioia, I.M., Maccacaro, T., 1989, "The Relation between X-ray Emission and Rotation in Late-Type Stars from the Perspective of X-ray Selection", Astrophys. J., 340, 10111023. Related online version (cited on 13 May 2004): http: //adsabs.harvard.edu/abs/1989ApJ . . 340.1011F 5.1

Florinski, V., Pogorelov, N.V., Zank, G.P., Wood, B.E., Cox, D.P., 2004, "On the Possibility of a Strong Magnetic Field in the Local Interstellar Medium", Astrophys. J., 604, 700-706. Related online version (cited on 13 May 2004):

http://adsabs.harvard.edu/abs/2004ApJ...604. .700F 4.2

Frisch, P.C., 1993, "G-star Astropauses: A Test for Interstellar Pressure", Astrophys. J., 407, 198-206. Related online version (cited on 13 May 2004):

http://adsabs.harvard.edu/abs/1993ApJ . .407 . .198F 4.3

Frisch, P.C., Slavin, J.D., 2003, "The Chemical Composition and Gas-to-Dust Mass Ratio of Nearby Interstellar Matter", Astrophys. J., 594, 844-858. Related online version (cited on 13 May 2004):

http://adsabs.harvard.edu/abs/2003ApJ. . 594. .844F 2.2

Gaidos, E.J., Güdel, M., Blake, G.A., 2000, "The Faint Young Sun Paradox: An Observational Test of an Alternative Solar Model", Geophys. Res. Lett., 27, 501-503 3, 5.2

Gayley, K.G., Zank, G.P., Pauls, H.L., Frisch, P.C., Welty, D.E., 1997, "One- versus Two-Shock Heliosphere: Constraining Models with Goddard High Resolution Spectrograph Lyman- $\alpha$ Spectra toward $\alpha$ Centauri", Astrophys. J., 487, 259-270. Related online version (cited on 13 May 2004):

http://adsabs.harvard.edu/abs/1997ApJ . . 487 . .259G 4.1, 4.2, 4.3, 4.3

Gough, D.O., 1981, "Solar Interior Structure and Luminosity Variations", Solar Phys., 74, 21-34. Related online version (cited on 13 May 2004):

http://adsabs.harvard.edu/abs/1981SoPh...74...21G 5.3

Grießmeier, J.-M., Stadelmann, A., Penz, T., Lammer, H., Selsis, F., Ribas, I., Guinan, E.F., Motschmann, U., Biernat, H.K., Weiss, W.W., 2004, "The Effect of Tidal Locking on the Magnetospheric and Atmospheric Evolution of 'Hot Jupiters"', Astron. Astrophys., 425, 753-762. Related online version (cited on 2 June 2007):

http://adsabs.harvard. edu/abs/2004A\%26A . .425 . 753GFUL 5.4

Living Reviews in Solar Physics

http://www. livingreviews.org/lrsp-2004-2 
Gringauz, K.I., Bezrukikh, V.V., Ozerov, V.D., Rybchinskii, R.E., 1962, "The Study of Interplanetary Ionized Gas, High-Energy Electrons and Corpuscular Radiation of the Sun, Employing Three-Electrode Charged Particle Traps on the Second Soviet Space Rocket", Planet. Space Sci., 9, 972.1

Güdel, M., 2004, "X-ray Astronomy of Stellar Coronae", Astron. Astrophys. Rev., 12, 71-237. Related online version (cited on 2 June 2007):

http://adsabs.harvard.edu/abs/2004A\%26ARv . .12 . .71G 1

Güdel, M., Guinan, E.F., Skinner, S.L., 1997, "The X-Ray Sun in Time: A Study of the Long-Term Evolution of Coronae of Solar-Type Stars", Astrophys. J., 483, 947-960. Related online version (cited on 13 May 2004):

http://adsabs.harvard.edu/abs/1997ApJ . . 483. .947G 5.1

Guinan, E.F., Ribas, I., Harper, G.M., 2003, "Far-Ultraviolet Emissions of the Sun in Time: Probing Solar Magnetic Activity and Effects on Evolution of Paleoplanetary Atmospheres", Astrophys. J., 594, 561-572. Related online version (cited on 13 May 2004): http://adsabs.harvard.edu/abs/2003ApJ . . .594. .561G 5.4

Gustafsson, B., Jørgensen, U.G., 1994, "Models of Late-Type Stellar Photospheres", Astron. Astrophys. Rev., 6, 19-65 1

Guzik, J.A., Willson, L.A., Brunish, W.M., 1987, "A Comparison between Mass-Losing and Standard Solar Models", Astrophys. J., 319, 957-965. Related online version (cited on 13 May 2004): http://adsabs.harvard.edu/abs/1987ApJ . . 319. .957G 5.3

Harper, G.M., Wood, B.E., Linsky, J.L., Bennett, P.D., Ayres, T.R., Brown, A., 1995, "A Semiempirical Determination of the Wind Velocity Structure for the Hybrid-Chromosphere Star $\alpha$ Trianguli Australis", Astrophys. J., 452, 407-422. Related online version (cited on 13 May 2004): http://adsabs.harvard.edu/abs/1995ApJ . . 452 . .407H 1

Hébrard, G., Mallouris, C., Ferlet, R., Koester, D., Lemoine, M., Vidal-Madjar, A., York, D., 1999, "Ultraviolet Observations of Sirius A and Sirius B with HST-GHRS: An Interstellar Cloud with a Possible Low Deuterium Abundance", Astron. Astrophys., 340, 643-658. Related online version (cited on 2 June 2007):

http://adsabs.harvard.edu/abs/1999A\%26A...350. .643H 4.2

Holzer, T.E., 1972, "Interaction of the Solar Wind with the Neutral Component of the Interstellar Gas", J. Geophys. Res., 77, 54072.3

Holzer, T.E., 1989, "Interaction between the Solar Wind and the Interstellar Medium", Annu. Rev. Astron. Astrophys., 27, 199-234. Related online version (cited on 13 May 2004): http://adsabs.harvard.edu/abs/1989ARA\%26A..27..199H 2.3

Holzwarth, V., Jardine, M., 2007, "Theoretical Mass Loss Rates of Cool Main-Sequence Stars", Astron. Astrophys., 463, 11-21. Related online version (cited on 2 June 2007): http: //adsabs.harvard.edu/abs/2007A\%26A..463...11HFUL 4.3

Hünsch, M., Schmitt, J.H.M.M., Sterzik, M.F., Voges, W., 1999, "The ROSAT All-Sky Survey Catalogue of the Nearby Stars", Astron. Astrophys. Suppl., 135, 319-338 2.1, 5.1

Izmodenov, V., Gloeckler, G., Malama, Y., 2003, "When Will Voyager 1 and 2 Cross the Termination Shock?", Geophys. Res. Lett., 30, 1351-1354 2.3 
Izmodenov, V., Alexashov, D., Myasnikov, A., 2005, "Direction of the Interstellar H Atom Inflow in the Heliosphere: Role of the Interstellar Magnetic Field", Astron. Astrophys., 437, L35-L38. Related online version (cited on 2 June 2007): http://adsabs.harvard.edu/abs/2005A\%26A. . 437L . 35IFUL 4.2

Izmodenov, V.V., Geiss, J., Lallement, R., Gloeckler, G., Baranov, V.B., Malama, Y.G., 1999a, "Filtration of Interstellar Hydrogen in the Two-Shock Heliospheric Interface: Inferences on the Local Interstellar Cloud Electron Density", J. Geophys. Res., 104, 4731-4742 2.2, 2.3

Izmodenov, V.V., Lallement, R., Malama, Y.G., 1999b, "Heliospheric and Astrospheric Hydrogen Absorption towards Sirius: No Need for Interstellar Hot Gas", Astron. Astrophys., 342, L13L16. Related online version (cited on 13 May 2004): http://adsabs.harvard.edu/abs/1999A\%26A...342L . .13I 4.1, 4.2

Izmodenov, V.V., Gruntman, M., Malama, Y.G., 2001, "Interstellar Hydrogen Atom Distribution Function in the Outer Heliosphere", J. Geophys. Res., 106, 10,681-10,690 2.3

Izmodenov, V.V., Wood, B.E., Lallement, R., 2002, "Hydrogen Wall and Heliosheath Lyman- $\alpha$ Absorption toward Nearby Stars: Possible Constraints on the Heliospheric Interface Plasma Flow", J. Geophys. Res., 107, 1308-1322 3, 4.2

Izmodenov, V.V., Malama, Y.G., Gloeckler, G., Geiss, J., 2004, "Filtration of Interstellar H, O, $\mathrm{N}$ Atoms through the Heliospheric Interface: Inferences on Local Interstellar Abundances of the Elements", Astron. Astrophys., 414, L29-L32 2.2

Jakosky, B.M., Phillips, R.J., 2001, "Mars' Volatile and Climate History", Nature, 412, 237-244 5.4

Jakosky, B.M., Pepin, R.O., Johnson, R.E., Fox, J.L., 1994, "Mars Atmospheric Loss and Isotopic Fractionation by Solar Wind Induced Sputtering and Photochemical Escape", Icarus, 111, 2712885.4

Johns-Krull, C.M., Valenti, J.A., 1996, "Detection of Strong Magnetic Fields on M Dwarfs", Astrophys. J. Lett., 459, L95-L98. Related online version (cited on 13 May 2004): http://adsabs.harvard.edu/abs/1996ApJ . . .459L . .95J 1

Kass, D.M., Yung, Y.L., 1995, "Loss of Atmosphere from Mars due to Solar Wind-Induced Sputtering", Science, 268, 697-699 5.4

Kasting, J.F., 1991, "CO² Condensation and the Climate of Early Mars", Icarus, 94, 1-13 5.3

Kasting, J.F., Ackerman, T.P., 1986, "Climatic Consequences of Very High Carbon Dioxide Levels in the Earth's Early Atmosphere", Science, 234, 1383-1385 5.3

Kirkman, D., Tytler, D., Suzuki, N., O’Meara, J.M., Lubin, D., 2003, "The Cosmological Baryon Density from the Deuterium-to-Hydrogen Ratio in QSO Absorption Systems: D/H toward Q1243+3047", Astrophys. J. Suppl. Ser., 149, 1-28. Related online version (cited on 13 May 2004): http://adsabs.harvard.edu/abs/2003ApJS..149...1K 4.1

Krimigis, S.M., Decker, R.B., Hill, M.E., Armstrong, T.P., Gloeckler, G., Hamilton, D.C., Lanzerotti, L.J., Roelof, E.C., 2003, "Voyager 1 Exited the Solar Wind at a Distance of 85AU from the Sun", Nature, 426, 45-48 2.3

Living Reviews in Solar Physics

http: //www. livingreviews.org/lrsp-2004-2 
Kruk, J.W., Howk, J.C., André, M., Moos, H.W., Oegerle, W.R., Oliveira, C., Sembach, K.R., Chayer, P., Linsky, J.L., Wood, B.E., Ferlet, R., Hébrard, G., Lemoine, M., Vidal-Madjar, A., Sonneborn, G., 2002, "Abundances of Deuterium, Nitrogen, and Oxygen toward HZ 43A: Results from the FUSE Mission", Astrophys. J. Suppl. Ser., 140, 19-36. Related online version (cited on 13 May 2004):

http://adsabs.harvard.edu/abs/2002ApJS. . 140...19K 4.2

Kudritzki, R.-P., Puls, J., 2000, "Winds from Hot Stars", Annu. Rev. Astron. Astrophys., 38, 613-666 1

Lallement, R., Bertin, P., 1992, "Northern-hemisphere observations of nearby interstellar gas: Possible detection of the local cloud", Astron. Astrophys., 266, 479-485. Related online version (cited on 13 May 2004):

http://adsabs.harvard.edu/abs/1992A\%26A ...266 . .479L 2.2, 4.3

Lallement, R., Ferlet, R., Lagrange, A.M., Lemoine, M., Vidal-Madjar, A., 1995, "Local Cloud structure from HST-GHRS", Astron. Astrophys., 304, 461-474. Related online version (cited on 13 May 2004):

http://adsabs.harvard.edu/abs/1995A\%26A . . 304 . .461L 2.2, 4.3

Lallement, R., Welsh, B.Y., Vergely, J.L., Crifo, F., Sfeir, D.M., 2003, "3D Mapping of the Dense Interstellar Gas around the Local Bubble", Astron. Astrophys., 411, 447-464 2.2, 2

Lammer, H., Stumptner, W., Molina-Cuberos, G.J., Bauer, S.J., Owen, T., 2000, "Nitrogen Isotope Fractionation and its Consequence for Titan's Atmospheric Evolution", Planet. Space Sci., 48, $529-5435.4$

Lammer, H., Lichtenegger, H.I.M., Kolb, C., Ribas, I., Guinan, E.F., Abart, R., Bauer, S.J., 2003, "Loss of Water from Mars: Implications for the Oxidation of the Soil", Icarus, 165, 9-25 5.4

Landsman, W.B., Henry, R.C., Moos, H.W., Linsky, J.L., 1984, "Observations of Interstellar Hydrogen and Deuterium toward Alpha Centauri A", Astrophys. J., 285, 801-807. Related online version (cited on 13 May 2004):

http://adsabs.harvard.edu/abs/1984ApJ . . 285 . .801L 4.1

Lazarus, A.J., McNutt Jr, R.L., 1990, "Plasma Observations in the Distant Heliosphere - A View from Voyager", in Physics of the Outer Heliosphere, (Eds.) Grzedzielski, S., Page, D.E., Proceedings of the 1st COSPAR Colloquium, held in Warsaw, Poland, 19-22 September 1989, vol. 1 of COSPAR Colloquia Series, pp. 229-234, Pergamon, Oxford, U.K.; New York, U.S.A. $2.1,5.1$

Lemoine, M., Vidal-Madjar, A., Hébrard, G., Désert, J.-M., Ferlet, R., Lecavelier des Étangs, A., Howk, J.C., André, M., Blair, W.P., Friedman, S.D., Kruk, J.W., Lacour, S., Moos, H.W., Sembach, K., Chayer, P., Jenkins, E.B., Koester, D., Linsky, J.L., Wood, B.E., Oegerle, W.R., Sonneborn, G., York, D.G., 2002, "Deuterium Abundance toward G191-B2B: Results from the FUSE Mission", Astrophys. J. Suppl. Ser., 140, 67-80. Related online version (cited on 13 May 2004):

http://adsabs.harvard.edu/abs/2002ApJS. .140 . .67L 4.2

Lim, J., White, S.M., 1996, "Limits to Mass Outflows from Late-Type Dwarf Stars", Astrophys. J. Lett., 462, L91-L94. Related online version (cited on 13 May 2004):

http://adsabs.harvard.edu/abs/1996ApJ ...462L. .91L 3 
Lim, J., White, S.M., Cully, S.L., 1996a, "The Eclipsing Radio Emission of the Precataclysmic Binary V471 Tauri", Astrophys. J., 461, 1009-1015. Related online version (cited on 13 May 2004):

http://adsabs.harvard.edu/abs/1996ApJ . .461.1009L 3

Lim, J., White, S.M., Slee, O.B., 1996b, "The Radio Properties of the dMe Flare Star Proxima Centauri", Astrophys. J., 460, 976-983. Related online version (cited on 13 May 2004):

http://adsabs.harvard.edu/abs/1996ApJ . . 460..976L 3

Linde, T., Gombosi, T.I., Roe, P.L., Powell, K.G., DeZeeuw, D.L., 1998, "Heliosphere in the Magnetized Local Interstellar Medium - Results of a Three-Dimensional MHD Simulation", J. Geophys. Res., 103, 1889-1904 4.2

Linsky, J.L., 1980, "Stellar Chromospheres", Annu. Rev. Astron. Astrophys., 18, 439-488. Related online version (cited on 13 May 2004):

http://adsabs.harvard.edu/abs/1980ARA\%26A. .18. .439L 1

Linsky, J.L., 1998, "Deuterium Abundance in the Local ISM and Possible Spatial Variations", Space Sci. Rev., 84, 285-296 2.2, 4.1, 4.1

Linsky, J.L., Wood, B.E., 1996, "The $\alpha$ Centauri Line of Sight: D/H Ratio, Physical Properties of Local Interstellar Gas, and Measurement of Heated Hydrogen (the "Hydrogen Wall") Near the Heliopause", Astrophys. J., 463, 254-270. Related online version (cited on 13 May 2004):

http://adsabs.harvard.edu/abs/1996ApJ . . 463 . 254L 5, 4.1, 4.1, 4.3, 4.3

Linsky, J.L., Brown, A., Gayley, K.G., Diplas, A., Savage, B.D., Ayres, T.R., Landsman, W.B., Shore, S.N., Heap, S.R., 1993, "Goddard High-Resolution Spectrograph Observations of the Local Interstellar Medium and the Deuterium/Hydrogen Ratio Along the Line of Sight toward Capella", Astrophys. J., 402, 694-709. Related online version (cited on 13 May 2004):

http://adsabs.harvard.edu/abs/1993ApJ . . 402 . .694L 4.1

Linsky, J.L., Diplas, A., Wood, B.E., Brown, A., Ayres, T.R., Savage, B.D., 1995, "Deuterium and the Local Interstellar Medium: Properties for the Procyon and Capella Lines of Sight", Astrophys. J., 451, 335-351. Related online version (cited on 13 May 2004):

http://adsabs.harvard.edu/abs/1995ApJ...451..335L 4.1

Linsky, J.L., Redfield, S., Wood, B.E., Piskunov, N., 2000, "The Three-dimensional Structure of the Warm Local Interstellar Medium. I. Methodology", Astrophys. J., 528, 756-766. Related online version (cited on 13 May 2004):

http://adsabs.harvard.edu/abs/2000ApJ ...528. .756L 2.2, 4.1

Lipatov, A.S., Zank, G.P., Pauls, H.L., 1998, "The Interaction of Neutral Interstellar H with the Heliosphere: A 2.5D Particle-Mesh Boltzmann Simulation", J. Geophys. Res., 103, 20,631$20,6422.3$

Lisse, C.M., Christian, D.J., Dennerl, K., Meech, K.J., Petre, R., Weaver, H.A., Wolk, S.J., 2001, "Charge Exchange-Induced X-Ray Emission from Comet C/1999 S4 (LINEAR)", Science, 292, $1343-13483$

Luhmann, J.G., Johnson, R.E., Zhang, M.H.G., 1992, "Evolutionary Impact of Sputtering of the Martian Atmosphere by $\mathrm{O}^{+}$Pickup Ions", Geophys. Res. Lett., 19, 2151-2154 5.4

Lundin, R., 2001, "Erosion by the Solar Wind", Science, 291, 19095.4

Living Reviews in Solar Physics

http://www. livingreviews.org/lrsp-2004-2 
MacGregor, K.B., Charbonneau, P., 1994, "Stellar Winds with Non-WKB Alfvén Waves I. Wind Models for Solar Coronal Conditions", Astrophys. J., 430, 387-398. Related online version (cited on 13 May 2004):

http://adsabs.harvard.edu/abs/1994ApJ...430. .387M 2.1

Malama, Y.G., Izmodenov, V.V., Chalov, S.V., 2006, "Modeling of the Heliospheric Interface: Multi-Component Nature of the Heliospheric Plasma", Astron. Astrophys., 445, 693-701. Related online version (cited on 2 June 2007):

http://adsabs.harvard.edu/abs/2006A\%26A..445. 693MFUL 4.2

McComas, D.J., Barraclough, B.L., Funsten, H.O., Gosling, J.T., Santiago-Muñoz, E., Skoug, R.M., Goldstein, B.E., Neugebauer, M., Riley, P., Balogh, A., 2000, "Solar Wind Observations Over Ulysses' First Full Polar Orbit", J. Geophys. Res., 105, 10,419-10,434 2.1, 1, 2.1

McComas, D.J., Elliott, H.A., von Steiger, R., 2002, "Solar Wind from High-Latitude Coronal Holes at Solar Maximum", Geophys. Res. Lett., 29, 28-31 2.1

McCullough, P.R., 1992, "The Interstellar Deuterium-to-Hydrogen Ratio: A Reevaluation of Lyman Absorption-Line Measurements", Astrophys. J., 390, 213-218. Related online version (cited on 13 May 2004):

http://adsabs.harvard.edu/abs/1992ApJ...390. .213M 4.1

McDonald, F.B., Stone, E.C., Cummings, A.C., Heikkila, B., Lal, N., Webber, W.R., 2003, "Enhancements of Energetic Particles near the Heliospheric Termination Shock", Nature, 426, 48-51 2.3

Mestel, L., 1984, "Angular Momentum Loss During Pre-Main Sequence Contraction", in Cool Stars, Stellar Systems, and the Sun, (Eds.) Baliunas, S.L., Hartmann, L., Proceedings of the Third Cambridge Workshop on Cool Stars, Stellar Systems, and the Sun, Held in Cambridge, Massachusetts, October 5-7, 1983, pp. 49-59, Pergamon, New York, U.S.A. 5.2, 5.2

Micela, G., Sciortino, S., Serio, S., Vaiana, G.S., Bookbinder, J., Golub, L., Harnden Jr, F.R., Rosner, R., 1985, "Einstein X-ray Survey of the Pleiades - The Dependence of X-ray Emission on Stellar Age", Astrophys. J., 292, 172-180. Related online version (cited on 13 May 2004): http://adsabs.harvard.edu/abs/1985ApJ . . 292. .172M 5.1

Minton, D.A., Malhotra, R., 2007, "Assessing the Massive Young Sun Hypothesis to Solve the Warm Young Earth Puzzle", Astrophys. J., 660, 1700-1706. Related online version (cited on 2 June 2007):

http://adsabs.harvard.edu/abs/2007ApJ...660.1700M 5.3

Moos, H.W., Sembach, K.R., Vidal-Madjar, A., York, D.G., Friedman, S.D., Hébrard, G., Kruk, J.W., Lehner, N., Lemoine, M., Sonneborn, G., Wood, B.E., Ake, T.B., André, M., Blair, W.P., Chayer, P., Gry, C., Dupree, A.K., Ferlet, R., Feldman, P.D., Green, J.C., Howk, J.C., Hutchings, J.B., Jenkins, E.B., Linsky, J.L., Murphy, E.M., Oegerle, W.R., Oliveira, C., Roth, K., Sahnow, D.J., Savage, B.D., Shull, J.M., Tripp, T.M., Weiler, E.J., Welsh, B.Y., Wilkinson, E., Woodgate, B.E., 2002, "Abundances of Deuterium, Nitrogen, and Oxygen in the Local Interstellar Medium: Overview of First Results from the FUSE Mission", Astrophys. J. Suppl. Ser., 140, 3-17. Related online version (cited on 13 May 2004): http://adsabs.harvard.edu/abs/2002ApJS. .140...3M 4.1, 4.1

Mullan, D.J., Sion, E.M., Bruhweiler, F.C., Carpenter, K.G., 1989, "Evidence for a Cool Wind from the K2 Dwarf in the Detached Binary V471 Tauri", Astrophys. J. Lett., 339, L33-L36. Related online version (cited on 13 May 2004): http://adsabs.harvard.edu/abs/1989ApJ... 339L . 33M 3 
Mullan, D.J., Doyle, J.G., Redman, R.O., Mathioudakis, M., 1992, "Limits on Detectability of Mass Loss from Cool Dwarfs", Astrophys. J., 397, 225-231. Related online version (cited on 13 May 2004):

http://adsabs.harvard.edu/abs/1992ApJ . . 397 . 225M 3

Mullan, D.J., Carpenter, K.G., Robinson, R.D., 1998, "Large Variations in the Winds of Single Cool Giants: $\lambda$ Velorum and $\gamma$ Crucis", Astrophys. J., 495, 927-932. Related online version (cited on 13 May 2004):

http://adsabs.harvard.edu/abs/1998ApJ . . .495. .927M 1

Müller, H.-R., Zank, G.P., Lipatov, A.S., 2000, "Self-Consistent Hybrid Simulations of the Interaction of the Heliosphere with the Local Interstellar Medium", J. Geophys. Res., 105, 27,419$27,4382.3,2.3,4.2$

Müller, H.-R., Zank, G.P., Wood, B.E., 2001a, "Modeling the Interstellar Medium-Stellar Wind Interactions of $\lambda$ Andromedae and $\epsilon$ Indi", Astrophys. J., 551, 495-506. Related online version (cited on 13 May 2004):

http://adsabs.harvard.edu/abs/2001ApJ...551..495M 4.3

Müller, H.-R., Zank, G.P., Wood, B.E., 2001b, "Modeling Stellar Wind Interaction with the ISM: Exploring Astrospheres and their Lyman- $\alpha$ Absorption", in The Outer Heliosphere: The Next Frontiers, (Eds.) Scherer, K., Fichtner, H., Fahr, H.J., Marsch, E., Proceedings of COSPAR Colloquium, Potsdam, Germany, July, 2001, vol. 11 of COSPAR Colloquia Series, pp. 53-56, Pergamon; Elsevier Science, New York, U.S.A.; Amsterdam, Netherlands 4.3

Murthy, J., Henry, R.C., Moos, H.W., Landsman, W.B., Linsky, J.L., Vidal-Madjar, A., Gry, C., 1987, "IUE Observations of Hydrogen and Deuterium in the Local Interstellar Medium", Astrophys. J., 315, 675-686. Related online version (cited on 13 May 2004):

http://adsabs.harvard.edu/abs/1987ApJ...315. .675M 4.1

Nerney, S., Suess, S.T., Schmahl, E.J., 1991, "Flow Downstream of the Heliospheric Terminal Shock - Magnetic Field Kinematics", Astron. Astrophys., 250, 556-564. Related online version (cited on 13 May 2004):

http://adsabs.harvard.edu/abs/1991A\%26A...250..556N 4.2

Neugebauer, M., Snyder, C., 1962, "Solar Plasma Experiment", Science, 138, 1095-1097 2.1

Opher, M., Liewer, P.C., Gombosi, T.I., Manchester, W., DeZeeuw, D.L., Sokolov, I., Toth, G., 2003, "Probing the Edge of the Solar System: Formation of an Unstable Jet Sheet", Astrophys. J. Lett., 591, L61-L65. Related online version (cited on 13 May 2004):

http: //adsabs.harvard.edu/abs/2003ApJ . .591L. .610 4.2

Opher, M., Liewer, P.C., Velli, M., Bettarini, L., Gombosi, T.I., Manchester, W., DeZeeuw, D.L., Sokolov, I., 2004, "Magnetic Effects at the Edge of the Solar System: MHD Instabilities, the de Laval Nozzle Effect and an Extended Jet", Astrophys. J., 611, 575-586 4.2

Opher, M., Stone, E.C., Liewer, P.C., 2006, "The Effects of a Local Interstellar Magnetic Field on Voyager 1 and 2 Observations", Astrophys. J. Lett., 640, L71-L74. Related online version (cited on 2 June 2007):

http://adsabs.harvard.edu/abs/2006ApJ . . 640L . .710 2.3, 4.2

Pallavicini, R., Golub, L., Rosner, R., Vaiana, G.S., Ayres, T., Linsky, J.L., 1981, "Relations among stellar X-ray emission observed from Einstein, stellar rotation and bolometric luminosity", Astrophys. J., 248, 279-290. Related online version (cited on 13 May 2004):

http://adsabs.harvard.edu/abs/1981ApJ . . 248. .279P 5.1

Living Reviews in Solar Physics

http://www.livingreviews.org/lrsp-2004-2 
Parker, E.N., 1958, "Dynamics of the Interplanetary Gas and Magnetic Fields", Astrophys. J., 128, 664-676. Related online version (cited on 13 May 2004):

http://adsabs.harvard.edu/abs/1958ApJ ...128. .664P 2.1

Parker, E.N., 1961, "The Stellar-Wind Regions", Astrophys. J., 134, 20-27. Related online version (cited on 13 May 2004):

http: //adsabs.harvard.edu/abs/1961ApJ . . 134_..20P 2.3

Parker, E.N., 1963, Interplanetary Dynamical Processes, vol. 8 of Interscience Monographs and Texts in Physics and Astronomy, Interscience Publishers, New York, U.S.A. 2.3

Pauls, H.L., Zank, G.P., 1997, "Interaction of a Nonuniform Solar Wind with the Local Interstellar Medium. 2. A Two-Fluid Model", J. Geophys. Res., 102, 19,779-19,788 4.2

Perez de Tejada, H., 1992, "Solar Wind Erosion of the Mars Early Atmosphere", J. Geophys. Res., 97, 3159-3167 5.4

Perryman, M.A.C., Lindegren, L., Kovalevsky, J., Hoeg, E., Bastian, U., Bernacca, P.L., Crézé, M., Donati, F., Grenon, M., van Leeuwen, F., van der Marel, H., Mignard, F., Murray, C.A., Le Poole, R.S., Schrijver, H., Turon, C., Arenou, F., Froeschlé, M., Petersen, C.S., 1997, "The Hipparcos Catalogue", Astron. Astrophys., 323, L49-L52. Related online version (cited on 13 May 2004):

http://adsabs.harvard.edu/abs/1997A\%26A . . 323L . .49P 4.3

Petit, P., Donati, J.-F., Aurière, M., Landstreet, J.D., Lignières, F., Marsden, S., Mouillet, D., Paletou, F., Toqué, N., Wade, G.A., 2005, "Large-Scale Magnetic Field of the G8 Dwarf $\xi$ Bootis A", Mon. Not. R. Astron. Soc., 361, 837-849. Related online version (cited on 2 June 2007): http://adsabs . harvard.edu/abs/2005MNRAS.361 . 837P 5.1

Pogorelov, N.V., Zank, G.P., Ogino, T., 2006, "Three-Dimensional Features of the Outer Heliosphere due to Coupling between the Interstellar and Interplanetary Magnetic Fields. II. The Presence of Neutral Hydrogen Atoms", Astrophys. J., 644, 1299-1316. Related online version (cited on 2 June 2007):

http://adsabs.harvard.edu/abs/2006ApJ...644.1299P 4.2

Prantzos, N., 1996, "The Evolution of D and ${ }^{3} \mathrm{He}$ in the Galactic Disk", Astron. Astrophys., 310, 106-114. Related online version (cited on 13 May 2004):

http://adsabs. harvard.edu/abs/1996A\%26A...310_.106P 4.1

Preusse, S., Kopp, A., Büchner, J., Motschmann, U., 2005, "Stellar Wind Regimes of Close-in Extrasolar Planets", Astron. Astrophys., 434, 1191-1200. Related online version (cited on 2 June 2007):

http://adsabs. harvard.edu/abs/2005A\%26A . .434.1191PFUL 5.4

Quémerais, E., Bertaux, J.-L., Lallement, R., Berthé, M., Kyrölä, E., Schmidt, W., 1999, "Interplanetary Lyman- $\alpha$ Line Profiles Derived from SWAN/SOHO Hydrogen Cell Measurements: The Full-sky Velocity Field", J. Geophys. Res., 104, 12,585-12,604 2.2

Quémerais, E., Bertaux, J.-L., Lallement, R., Berthé, M., Kyrölä, E., Schmidt, W., 2000, "SWAN/SOHO H Cell Measurements: The First Year", Adv. Space Res., 26, 815-818 2.2

Ratkiewicz, R., Barnes, A., Molvik, G.A., Spreiter, J.R., Stahara, S.S., Vinokur, M., Venkateswaran, S., 1998, "Effect of Varying Strength and Orientation of Local Interstellar Magnetic Field on Configuration of Exterior Heliosphere: 3D MHD Simulations", Astron. Astrophys., 335, 363-369. Related online version (cited on 13 May 2004): http://adsabs.harvard.edu/abs/1998A\%26A . . 335..363R 4.2 
Redfield, S., Linsky, J.L., 2000, "The Three-dimensional Structure of the Warm Local Interstellar Medium. II. The Colorado Model of the Local Interstellar Cloud", Astrophys. J., 534, 825-837. Related online version (cited on 13 May 2004): http://adsabs.harvard.edu/abs/2000ApJ...534. .825R 2.2, 4.1

Redfield, S., Linsky, J.L., 2001, "Microstructure of the Local Interstellar Cloud and the Identification of the Hyades Cloud", Astrophys. J., 551, 413-428. Related online version (cited on 13 May 2004): http://adsabs.harvard.edu/abs/2001ApJ...551..413R 2.2

Ribas, I., Guinan, E.F., Güdel, M., Audard, M., 2005, "Evolution of the Solar Activity over Time and Effects on Planetary Atmospheres. I. High-Energy Irradiances (1-1700 ̊)", Astrophys. J., 622, 680-694. Related online version (cited on 2 June 2007):

http: //adsabs.harvard.edu/abs/2005ApJ. . .622 . 680R 5.4

Sackmann, I.-J., Boothroyd, A.I., 2003, "Our Sun. V. A Bright Young Sun Consistent with Helioseismology and Warm Temperatures on Ancient Earth and Mars", Astrophys. J., 583, 10241039. Related online version (cited on 13 May 2004):

http://adsabs.harvard.edu/abs/2003ApJ. . 583.1024S 5.3

Sagan, C., Mullen, G., 1972, "Earth and Mars: Evolution of Atmospheres and Surface Temperatures", Science, 177, 52-56 5.3

Schmitt, J.H.M.M., 1997, "Coronae on Solar-Like Stars", Astron. Astrophys., 318, 215-230. Related online version (cited on 13 May 2004):

http://adsabs.harvard.edu/abs/1997A\%26A...318. .215S 2.1

Schmitt, J.H.M.M., Liefke, C., 2004, "NEXXUS: A Comprehensive ROSAT Survey of Coronal Xray Emission Among Nearby Solar-like Stars", Astron. Astrophys., 417, 651-665. Related online version (cited on 2 June 2007):

http://adsabs.harvard.edu/abs/2004A\%26A . .417 .651SFUL 5.1

Schrijver, C.J., Title, A.M., 2001, "On the Formation of Polar Spots in Sun-like Stars", Astrophys. J., 551, 1099-1106. Related online version (cited on 2 June 2007): http://adsabs.harvard.edu/abs/2001ApJ. ..551.1099S 5.1

Schrijver, C.J., DeRosa, M.L., Title, A.M., 2003, "Asterospheric Magnetic Fields and Winds of Cool Stars", Astrophys. J., 590, 493-501. Related online version (cited on 13 May 2004): http: //adsabs.harvard.edu/abs/2003ApJ . .590..493S 1, 5.1

Sfeir, D.M., Lallement, R., Crifo, F., Welsh, B.Y., 1999, "Mapping the Contours of the Local bubble: Preliminary Results", Astron. Astrophys., 346, 785-797. Related online version (cited on 13 May 2004):

http://adsabs.harvard.edu/abs/1999A\%26A...346..785S 2.2

Shaviv, N.J., 2003, "Toward a Solution to the Early Faint Sun Paradox: A Lower Cosmic Ray Flux from a Stronger Solar Wind", J. Geophys. Res., 108, 1437-1444 5.3

Skumanich, A., 1972, "Time Scales for Ca II Emission Decay, Rotational Braking, and Lithium Depletion", Astrophys. J., 171, 565-567. Related online version (cited on 13 May 2004):

http://adsabs.harvard.edu/abs/1972ApJ...171..565S 1, 5.1

Slavin, J.D., Frisch, P.C., 2002, "The Ionization of Nearby Interstellar Gas", Astrophys. J., 565, 364-379. Related online version (cited on 13 May 2004):

http://adsabs.harvard.edu/abs/2002ApJ...565..364S 2.2

Living Reviews in Solar Physics

http://www. livingreviews . org/lrsp-2004-2 
Snowden, S.L., Freyberg, M.J., Plucinsky, P.P., Schmitt, J.H.M.M., Truemper, J., Voges, W., Edgar, R.J., McCammon, D., Sanders, W.T., 1995, "First Maps of the Soft X-Ray Diffuse Background from the ROSAT XRT/PSPC All-Sky Survey", Astrophys. J., 454, 643-653. Related online version (cited on 13 May 2004):

http://adsabs.harvard.edu/abs/1995ApJ...454.643S 2.2

Soderblom, D.R., Stauffer, J.R., MacGregor, K.B., Jones, B.F., 1993, "The Evolution of Angular Momentum Among Zero-Age Main-Sequence Solar-Type Stars", Astrophys. J., 409, 624-634. Related online version (cited on 13 May 2004): http://adsabs.harvard.edu/abs/1993ApJ . . 409. .624S 5.1

Stauffer, J.R., Caillault, J.-P., Gagné, M., Prosser, C.F., Hartmann, L.W., 1994, “A Deep Imaging Survey of the Pleiades with ROSAT", Astrophys. J. Suppl. Ser., 91, 625-657. Related online version (cited on 13 May 2004):

http: //adsabs.harvard.edu/abs/1994ApJS . .91. .625S 5.1

Stepień, K., 1988, "Spin-Down of Cool Stars During their Main-Sequence Life", Astrophys. J., 335, 907-913. Related online version (cited on 13 May 2004):

http://adsabs.harvard.edu/abs/1988ApJ...335. .907S 5.2

Stern, D.P., 1989, "A Brief History of Magnetospheric Physics Before the Spaceflight Era", Rev. Geophys., 27, 103-114. Related online version (cited on 25 March 2004): http://www.phy6.org/Education/Intro.html 2.1

Stone, E.C., Cummings, A.C., McDonald, F.B., Heikkila, B.C., Lal, N., Webber, W.R., 2005, "Voyager 1 Explores the Termination Shock Region and the Heliosheath Beyond", Science, 309, 2017-2020. Related online version (cited on 2 June 2007): http://adsabs.harvard.edu/abs/2005Sci...309.2017S 2.3

Strassmeier, K.G., 2002, "Doppler Images of Starspots", Astron. Nachr., 323, 309-316. Related online version (cited on 2 June 2007):

http://adsabs.harvard.edu/abs/2002AN . . . 323. .309S 5.1

Suess, S.T., 1990, "The Heliopause", Rev. Geophys., 28, 97-115 2.3

Svensmark, H., 1988, "Influence of Cosmic Rays on Earth's Climate", Phys. Rev. Lett., 81, 502750305.3

Toner, C.G., Gray, D.F., 1988, "The Starpatch on the G8 Dwarf $\xi$ Bootis A", Astrophys. J., 334, 1008-1020. Related online version (cited on 2 June 2007):

http://adsabs.harvard.edu/abs/1988ApJ . . .334.1008T 5.1

Tosi, M., Steigman, G., Matteucci, F., Chiappini, C., 1998, "Is High Primordial Deuterium Consistent with Galactic Evolution?", Astrophys. J., 498, 226-235. Related online version (cited on 13 May 2004):

http://adsabs.harvard.edu/abs/1998ApJ...498. .226T 4.1

van den Oord, G.H.J., Doyle, J.G., 1997, "Constraints on Mass Loss from dMe Stars: Theory and Observations", Astron. Astrophys., 319, 578-588. Related online version (cited on 13 May 2004):

http://adsabs. harvard.edu/abs/1997A\%26A . .319. .578V 3

Vidal-Madjar, A., Ferlet, R., 2002, "Hydrogen Column Density Evaluations toward Capella: Consequences on the Interstellar Deuterium Abundance", Astrophys. J. Lett., 571, L169-L172. Related online version (cited on 13 May 2004):

http://adsabs.harvard.edu/abs/2002ApJ...571L.169V 4.2 
Vogt, S.S., Penrod, G.D., Hatzes, A.P., 1987, "Doppler images of rotating stars using maximum entropy image reconstruction", Astrophys. J., 321, 496-515. Related online version (cited on 13 May 2004):

http://adsabs.harvard.edu/abs/1987ApJ . . .321. .496V 1

Walker, J.C.G., 1985, "Carbon Dioxide on the Early Earth", Origins of Life, 16, 117-127 5.3

Wallis, M., 1975, "Local Interstellar Medium", Nature, 254, 207-127 2.3

Walter, F.M., 1982, "On the Coronae of Rapidly Rotating Stars. III. An Improved Coronal Rotation-Activity Relation in Late-Type Dwarfs", Astrophys. J., 253, 745-751. Related online version (cited on 13 May 2004):

http://adsabs.harvard.edu/abs/1982ApJ . . .253. .745W 5.1

Walter, F.M., 1983, "On the Coronae of Rapidly Rotating Stars. IV. Coronal Activity in F Dwarfs and Implications for the Onset of the Dynamo", Astrophys. J., 274, 794-800. Related online version (cited on 13 May 2004): http://adsabs.harvard.edu/abs/1983ApJ . . 274 . .794W 5.1

Wargelin, B.J., Drake, J.J., 2002, "Stringent X-Ray Constraints on Mass Loss from Proxima Centauri", Astrophys. J., 578, 503-514. Related online version (cited on 13 May 2004): http://adsabs.harvard.edu/abs/2002ApJ...578. .503W 3

Weber, E.J., Davis Jr, L., 1967, "The Angular Momentum of the Solar Wind", Astrophys. J., 148, 217-228. Related online version (cited on 13 May 2004): http://adsabs.harvard.edu/abs/1967ApJ...148..217W 5.2

Witte, M., Rosenbauer, H., Banaszkewicz, M., Fahr, H.J., 1993, "The ULYSSES Neutral Gas Experiment - Determination of the Velocity and Temperature of the Interstellar Neutral Helium", Adv. Space Res., 13, 121-130 2.2

Witte, M., Rosenbauer, H., Banaszkewicz, M., 1996, "Recent Results on the Parameters of the Interstellar Helium from the Ulysses/Gas Experiment", Space Sci. Rev., 78, 289-296 2.2

Wood, B.E., Linsky, J.L., 1997, "A New Measurement of the Electron Density in the Local Interstellar Medium”, Astrophys. J. Lett., 474, L39-L42. Related online version (cited on 13 May 2004):

http://adsabs.harvard.edu/abs/1997ApJ...474L. .39W 2.2

Wood, B.E., Linsky, J.L., 1998, "The Local ISM and its Interaction with the Winds of Nearby Late-Type Stars", Astrophys. J., 492, 788-803. Related online version (cited on 13 May 2004): http://adsabs.harvard.edu/abs/1998ApJ . .492. .788W 2.2, 4.3

Wood, B.E., Alexander, W.R., Linsky, J.L., 1996, "The Properties of the Local Interstellar Medium and the Interaction of the Stellar Winds of $\epsilon$ Indi and $\lambda$ Andromedae with the Interstellar Environment", Astrophys. J., 470, 1157-1171. Related online version (cited on 13 May 2004): http: //adsabs.harvard.edu/abs/1996ApJ . . .470.1157W 4.3, 4.3

Wood, B.E., Linsky, J.L., Zank, G.P., 2000a, "Heliospheric, Astrospheric, and Interstellar Ly $\alpha$ Absorption toward 36 Ophiuchi", Astrophys. J., 537, 304-311. Related online version (cited on 13 May 2004):

http://adsabs.harvard.edu/abs/2000ApJ . .537. .304W 4.2, 4.3

Living Reviews in Solar Physics

http: //www . livingreviews . org/lrsp-2004-2 
Wood, B.E., Müller, H.-R., Zank, G.P., 2000b, "Hydrogen Lyman- $\alpha$ Absorption Predictions by Boltzmann Models of the Heliosphere", Astrophys. J., 542, 493-503. Related online version (cited on 13 May 2004):

http://adsabs.harvard.edu/abs/2000ApJ...542. .493W 4, 2.3, 4.1, 8, 4.2

Wood, B.E., Linsky, J.L., Müller, H.-R., Zank, G.P., 2001, "Observational Estimates for the MassLoss Rates of $\alpha$ Centauri and Proxima Centauri Using Hubble Space Telescope Ly $\alpha$ Spectra", Astrophys. J. Lett., 547, L49-L52. Related online version (cited on 13 May 2004): http://adsabs.harvard.edu/abs/2001ApJ ...547L. .49W 3, 4.1, 7, 4.3, 4.3, 9

Wood, B.E., Müller, H.-R., Zank, G.P., Linsky, J.L., 2002, "Measured Mass-Loss Rates of Solar-like Stars as a Function of Age and Activity", Astrophys. J., 574, 412-425. Related online version (cited on 13 May 2004):

http: //adsabs. harvard.edu/abs/2002ApJ . . .574. .412W 4.3, 4.3, 10, 12, 4.3

Wood, B.E., Linsky, J.L., Müller, H.-R., Zank, G.P., 2003a, "A Search for Ly $\alpha$ emission from the Astrosphere of 40 Eridani A", Astrophys. J., 591, 1210-1219. Related online version (cited on 13 May 2004):

http://adsabs.harvard.edu/abs/2003ApJ ...591.1210W 4.3

Wood, B.E., Redfield, S., Linsky, J.L., 2003b, "The 3D-Structure of the LISM", in The Interstellar Environment of the Heliosphere, (Eds.) Breitschwerdt, D., Haerendel, G., International Colloquium in Honour of Stanislaw Grzedzielski, Paris 2001, vol. 285 of MPE Report, pp. 25-47, MPE, Garching, Germany. Related online version (cited on 13 May 2004): http://arXiv.org/abs/astro-ph/0107033 2.2, 6

Wood, B.E., Linsky, J.L., Hébrard, G., Williger, G.M., Moos, H.W., Blair, W.P., 2004, "Two New Low Galactic D/H Measurements from the Far Ultraviolet Spectroscopic Explorer (FUSE)", Astrophys. J., 609, 838-853. Related online version (cited on 13 May 2004):

http://adsabs. harvard.edu/abs/2004ApJ . .609. . 838W 4.1, 4.1

Wood, B.E., Müller, H.-R., Zank, G.P., Linsky, J.L., Redfield, S., 2005a, "New Mass-Loss Measurements from Astrospheric Lya Absorption", Astrophys. J. Lett., 628, L143-L146. Related online version (cited on 2 June 2007):

http://adsabs.harvard.edu/abs/2005ApJ ...628L.143W 4.3, 4.3, 11, 13, 4.3, 14, 5.1, 5.1, 15

Wood, B.E., Redfield, S., Linsky, J.L., Müller, H.-R., Zank, G.P., 2005b, "Stellar Ly $\alpha$ Emission Lines in the Hubble Space Telescope Archive: Intrinsic Line Fluxes and Absorption from the Heliosphere and Astrospheres", Astrophys. J. Suppl. Ser., 159, 118-140. Related online version (cited on 2 June 2007):

http://adsabs.harvard.edu/abs/2005ApJS. .159. .118W 4.2, 4.3

Wood, B.E., Izmodenov, V.V., Linsky, J.L., Alexashov, D., 2007a, "Dependence of Heliospheric Ly $\alpha$ Absorption on the Interstellar Magnetic Field", Astrophys. J., 659, 1784-1791. Related online version (cited on 2 June 2007):

http://adsabs.harvard.edu/abs/2007ApJ...659.1784W 4.2

Wood, B.E., Izmodenov, V.V., Linsky, J.L., Malama, Y.G., 2007b, "Ly $\alpha$ Absorption from Heliosheath Neutrals", Astrophys. J., 657, 609-617. Related online version (cited on 2 June 2007): http://adsabs.harvard.edu/abs/2007ApJ ..657..609W 4.2, 4.2

York, D.G., Rogerson, J.B., 1976, "The Abundance of Deuterium Relative to Hydrogen in Interstellar Space", Astrophys. J., 203, 378-385. Related online version (cited on 13 May 2004): http://adsabs. harvard.edu/abs/1976ApJ . . 203. .378Y 4.1 
Zank, G.P., 1999, "Interaction of the Solar Wind with the Local Interstellar Medium: A Theoretical Perspective", Space Sci. Rev., 89, 413-688 2.3

Zank, G.P., Pauls, H.L., Williams, L.L., Hall, D.T., 1996, "Interaction of the Solar Wind with the Local Interstellar Medium: A Multifluid Approach", J. Geophys. Res., 101, 21,639-21,656 2.3, $2.3,4.2$ 